\title{
Stabilized finite element method for the stationary mixed Stokes-Darcy problem
}

\author{
Jiaping Yu ${ }^{1}$, Md. Abdullah Al Mahbub ${ }^{2,3}$, Feng Shi ${ }^{4 *}$ and Haibiao Zheng ${ }^{2,3}$
}

\author{
"Correspondence: \\ shi.feng@hit.edu.cn \\ ${ }^{4}$ College of Science, Harbin Institute \\ of Technology, Shenzhen, China \\ Full list of author information is \\ available at the end of the article
}

\begin{abstract}
This paper considers numerical methods for solving the viscous incompressible steady-state Stokes-Darcy problem that can be implemented by the use of existing surface water and groundwater codes. In the porous medium problem for subsurface flow, a mixed discretization, which describes the macroscopic properties of a filtration process and is vigorous with respect to the variations in the material data, is often advocated. However, the theory of mixed spacial discretizations to Stokes-Darcy problems is far less developed than non-mixed versions. We develop herein a new robust stabilized fully mixed discretization technique in the porous media region coupled with the fluid region via the physically appropriate coupling conditions on the interface. The method developed here does not use any Lagrange multiplier and introduces a stabilization term in the temporal discretization to ensure the stability of the finite element scheme. The well-posedness of the finite element scheme and its convergence analysis are also derived. Finally, the efficiency and accuracy of the numerical methods are illustrated by several testing examples.
\end{abstract}

Keywords: Stokes-Darcy problem; Mixed finite element; Free flow; Porous media flow; Stabilized scheme

\section{Introduction}

Many important applications require accurate solution of multi-domain, multi-physics coupling of groundwater and surface flows. The Stokes-Darcy fluid flow model is a coupling among surface and subsurface flows, which occurs in many natural and industrial events such as biomedicine, industrial processes, blood flow motion in the arteries, ground water fluid flow in a karst aquifer, biofluid dynamics, hydrology, contaminant transport in a groundwater geometry through rivers, industrial filtration, petroleum engineering, spontaneous combustion of coal stockpiles, and so on [1-5]. A dynamical fluid model describes the free fluid flow in the conduit and porous medium fluid flow in the matrix separated by a shared interface.

Coupling two separate models via an interface generally needs some appropriate and effective interface conditions. For Stokes-Darcy model, the coupling conditions are well studied and usually modeled by three interface conditions. The first two consist of the continuity of the normal velocity across the interface, which is a consequence of the conservation of mass, and the balance of normal force across the interface. The third interface condition is a benchmark boundary condition invented by Beavers-Joseph in 1967 by conducting several experiments [6]. In the Beavers-Joseph interface condition, the tangential

(c) The Author(s) 2018. This article is distributed under the terms of the Creative Commons Attribution 4.0 International License (http://creativecommons.org/licenses/by/4.0/), which permits unrestricted use, distribution, and reproduction in any medium, provided you give appropriate credit to the original author(s) and the source, provide a link to the Creative Commons license, and indicate if changes were made. 
component of the stress force of the flow in the free medium at the interface is proportional to the jump of the tangential velocity across the interface. Saffman modified this interface condition in [7], and found that the velocity of the porous medium is smaller and can be dropped. Jones [8] reinterpreted this law for application to the curved boundaries and non-tangential flows.

After the rigorous investigation of Beavers and Joseph on the coupling condition between conduit and matrix, the study of Stokes-Darcy fluid flow model has become one of the most attractive research topics. The study of Stokes-Darcy fluid flow model started with intense numerical implementation $[9,10]$ while theoretical analysis begun with $[11$, 12]. The equilibrium Stokes-Darcy problem has only been understood recently in [1114], and there has been an intense development of extensions and refinements thereafter. Over the last few decades, a great deal of effort has been devoted to developing an appropriate approximate solution to study the Stokes-Darcy model. Many different techniques and numerical methods were applied to investigate the Stokes-Darcy fluid flow model, such as coupled finite elements methods [15-21], two-grid/multi-grid methods [22-28], discontinuous Galerkin finite element methods [29-32], partitioned time stepping methods [33-36], least squares methods [37-39], domain decomposition methods [1, 2, 4, 40-48], local-parallel finite element methods [49], interface relaxation method [50, 51], motar finite element methods [52, 53], Lagrange multiplier methods [11, 54-59]. Among them, the theory and research literature about mixed spatial discretizations had been far less developed than that of non-mixed versions.

In [11], Layton, Schieweck and Yotov investigated a mixed variational formulation in both domains based on Beavers-Joseph-Saffman interface conditions and utilized a Lagrange multiplier on the interface to prove the well-posedness of the weak solution, which allowed us to decouple the coupled problem into two subproblems. Discacciati et al. studied the Navier/Stokes-Darcy fluid flow model and proposed an iterative subdomain method by using continuous finite elements in both regions with a second order elliptic problem in the Darcy domain and a standard mixed element method in the Stokes domain in $[43,47]$. A locally conservative numerical method was applied to investigate the coupled free and porous medium flow by Rivière et al. in [30,31], where a mixed finite element method was used for Darcy domain and a discontinuous Galerkin finite element method for Stokes region. A study of porous medium with small and large cavities using mixed finite element couple with the vuggy medium on the microscopic scale using Stokes equations was performed by Arbogast et al. in [15]. Mu and Xu studied mixed StokesDarcy fluid flow model using the two-grid method in [22]. A unified stabilized method was studied by Burman et al. [60], where they consider the lowest possible approximation order. A preconditioning technique was applied in [61] for mixed Stokes-Darcy fluid flow model by Cai et al. In [57-59], Gatica et al. extended the work of Layton [11] in a new dimension considering conforming mixed finite elements where the matrix subdomain is completely enclosed by the conduit region. The interface conditions allowed the introduction of the trace of the porous medium pressure as a suitable Lagrange multiplier. The finite element subspaces defining the discrete formulation employ Bernardi-Raugel and Raviart-Thomas elements for the velocities, piecewise constants for the pressures and continuous piecewise-linear elements for the Lagrange multiplier. For more work on the mixed formulation, the reader can check [62-66]. 
In this paper, we investigate an approximate solution of the stationary Stokes-Darcy problem by developing a fully mixed stabilized finite element technique. The mixed formulation discussed in $[11,57-59,62,63]$ essentially needs a Lagrange multiplier, and the implementation is not easy in order to derive stability and convergence analysis. The fully mixed finite element scheme is proposed herein without introducing any Lagrange multiplier and computation is straightforward. A fully-discrete finite element algorithm is proposed, and we introduce a stabilized term essentially to ensure the stability of the temporal discretization. The stability of the finite element scheme is derived and the convergence analysis is discussed. To show the validity and efficiency of the numerical methods, we perform two numerical experiments which confirm the optimal convergence order considering an exact solution of the model problem. The effects of the stabilization parameter are investigated by considering different values of the stabilization parameter, which helps us to choose an accurate value of the stabilization parameter to obtain the optimal convergence order.

The rest of the paper is organized as follows. In Sect. 2, we describe the well known Stokes-Darcy fluid flow model with interface conditions. In Sect. 3, we present some notations, preliminaries, and variational formulation. The stabilized finite element method and its stability are discussed in Sect. 4. Section 5 contains a convergence analysis of the finite element scheme. In Sect. 6, we present two numerical tests to show the accuracy of the numerical methods. Finally, we conclude with a summary in Sect. 7.

\section{The model problem}

Let the two bounded domains be denoted by $\Omega_{f}, \Omega_{p} \subset R^{d}(d=2$ or 3$)$ and lie across an interface $\Gamma$ from each other. Here $\Omega_{f} \cap \Omega_{p}=\emptyset$, and $\bar{\Omega}_{f} \cap \bar{\Omega}_{p}=\Gamma, \bar{\Omega}=\bar{\Omega}_{f} \cup \bar{\Omega}_{p}$, $\mathbf{n}_{f}$ and $\mathbf{n}_{p}$ are the unit outward normal vectors on $\partial \Omega_{f}$ and $\partial \Omega_{p}$, respectively, and $\boldsymbol{\tau}_{i}, i=1, \ldots, d-1$, are the unit tangential vectors on the interface $\Gamma, \Gamma_{f}=\partial \Omega_{f} \backslash \Gamma, \Gamma_{p}=\partial \Omega_{p} \backslash \Gamma$. Note that $\mathbf{n}_{p}=-\mathbf{n}_{f}$ on $\Gamma$. Figure 1 shows a sketch of the problem domain, its boundaries and some other notations.

The fluid velocity and pressure $\mathbf{u}_{f}(x)$ and $p(x)$ are governed by the Stokes equation in $\Omega_{f}$ :

$$
\begin{aligned}
& -\nabla \cdot \mathbb{T}=-2 v \nabla \cdot \mathbb{D}\left(\mathbf{u}_{f}\right)+\nabla p=\mathbf{f}_{f} \quad \text { in } \Omega_{f}, \\
& \nabla \cdot \mathbf{u}_{f}=0 \quad \text { in } \Omega_{f},
\end{aligned}
$$

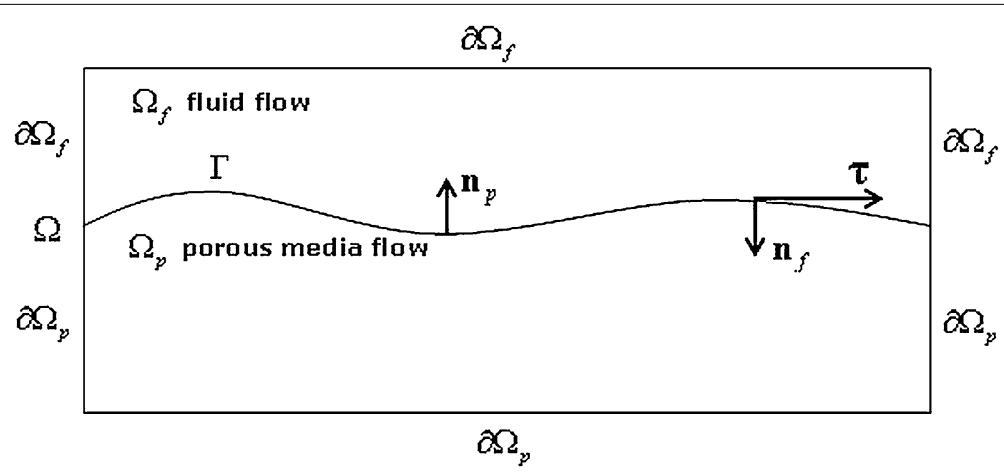

Figure 1 Global domain $\Omega$ consisting of the fluid region $\Omega_{f}$ and the porous media region $\Omega_{p}$ separated by the interface $\Gamma$ 
where $\mathbb{T}=-p \mathbb{I}+2 v \mathbb{D}\left(\mathbf{u}_{f}\right)$ denotes the stress tensor, and $\mathbb{D}\left(\mathbf{u}_{f}\right)=\frac{1}{2}\left(\nabla \mathbf{u}_{f}+\left(\nabla \mathbf{u}_{f}\right)^{T}\right)$ represents the deformation tensor. The porous media flow is governed by the following Darcy equations on $\Omega_{p}$ through the fluid velocity $\mathbf{u}_{p}(x)$ and the piezometric head $\phi(x)$ :

$$
\begin{aligned}
& \mathbf{u}_{p}=-\mathbf{K} \nabla \phi \quad \text { in } \Omega_{p}, \\
& \nabla \cdot \mathbf{u}_{p}=f_{p} \quad \text { in } \Omega_{p} .
\end{aligned}
$$

We impose impermeable boundary conditions, $\mathbf{u}_{p} \cdot \mathbf{n}_{p}=0$ on $\Gamma_{p}$, on the exterior boundary of the porous media region, and no slip conditions, $\mathbf{u}_{f}=0$ on $\Gamma_{f}$, in the Stokes region. Both selections of boundary conditions can be modified. On $\Gamma$ the interface coupling conditions are conservation of mass, balance of forces and a tangential condition on the fluid region's velocity on the interface. The correct tangential condition is not completely understood (possibly due to matching a pointwise velocity in the fluid region with an averaged or homogenized velocity in the porous region). In this paper, we take the Beavers-JosephSaffman (-Jones), see [6-8, 13], interfacial coupling

$$
\begin{aligned}
& \mathbf{u}_{f} \cdot \mathbf{n}_{f}+\mathbf{u}_{p} \cdot \mathbf{n}_{p}=0 \quad \text { on } \Gamma, \\
& -\mathbf{n}_{f} \cdot \mathbb{T} \cdot \mathbf{n}_{f}=p-2 v \mathbf{n}_{f} \cdot \mathbb{D}\left(\mathbf{u}_{f}\right) \cdot \mathbf{n}_{f}=\rho g \phi \quad \text { on } \Gamma, \\
& -\mathbf{n}_{f} \cdot \mathbb{T} \cdot \tau_{i}=-2 \mathbf{n}_{f} \cdot \mathbb{D}\left(\mathbf{u}_{f}\right) \cdot \tau_{i}=\frac{\alpha}{\sqrt{\tau_{i} \cdot \mathbf{K} \tau_{i}}} \mathbf{u}_{f} \cdot \tau_{i}, \quad 1 \leq i \leq(d-1) \text { on } \Gamma .
\end{aligned}
$$

This is a simplification of the original and more physically realistic Beavers-Joseph conditions (in which $\mathbf{u}_{f} \cdot \tau_{i}$ in (2.8) is replaced by $\left(\mathbf{u}_{f}-\mathbf{u}_{p}\right) \cdot \tau_{i}$ ); see [6]. Here we denote

$\mathbf{f}_{f}, f_{p}$ - body forces in the fluid region and source in the porous region,

K - symmetric positive definite (SPD) hydraulic conductivity tensor,

$v$ - kinematic viscosity of fluid,

$\alpha$ - constant parameter.

We shall also assume that all material and fluid parameters defined above are uniformly positive and bounded, i.e.,

$$
0 \leq k_{\min } \leq \lambda(\mathbf{K}) \leq k_{\max }<\infty
$$

\section{Notations and the variational formulation}

In this part, we first introduce some Sobolev spaces [67] and norms. We denote the usual $L^{2}$ norm by $\|\cdot\|$ for square integrable scalar/vector/matrix-valued functions defined on domain $\Omega_{f}$ or $\Omega_{p}$, and the corresponding inner product by (., .), and similarly the $L^{2}$-norm in $L^{2}(\Gamma)$ by $\|\cdot\|_{\Gamma}$, for instance,

$$
\begin{aligned}
& \|p\|:=\left(\sum_{i=1}^{d} \int_{\Omega_{f}}|p|^{2} \mathrm{~d} x\right)^{1 / 2}, \quad \text { for } p \in L^{2}\left(\Omega_{f}\right), \\
& \left\|\mathbf{v}_{f}\right\|:=\left(\sum_{i=1}^{d}\left\|v_{f}^{i}\right\|^{2}\right)^{1 / 2}, \quad \text { for } \mathbf{v}_{f}=\left(v_{f}^{1}, \ldots, v^{d}\right) \in L^{2}\left(\Omega_{f}\right)^{d},
\end{aligned}
$$




$$
\left\|\nabla \mathbf{v}_{f}\right\|:=\left(\sum_{i=1}^{d}\left\|\nabla v_{f}^{i}\right\|^{2}\right)^{1 / 2}, \quad \text { for } \mathbf{v}_{f}=\in L^{2}\left(\Omega_{f}\right)^{d}
$$

and the inner product over $\Gamma$ by

$$
\left(\phi_{1}, \phi_{2}\right)_{\Gamma}=\int_{\Gamma} \phi_{1} \phi_{2} \mathrm{~d} \Gamma
$$

By setting the space

$$
H_{\mathrm{div}}=H\left(\operatorname{div} ; \Omega_{p}\right):=\left\{\mathbf{v}_{p} \in L^{2}\left(\Omega_{p}\right)^{d}: \nabla \cdot \mathbf{v}_{p} \in L^{2}\left(\Omega_{p}\right)\right\},
$$

we introduce the following spaces:

$$
\begin{aligned}
& \mathbf{X}_{f}:=\left\{\mathbf{v}_{f} \in H^{1}\left(\Omega_{f}\right)^{d}: \mathbf{v}_{f}=0 \text { on } \Gamma_{f}\right\}, \\
& Q_{f}:=L^{2}\left(\Omega_{f}\right), \\
& \mathbf{X}_{p}:=\left\{\mathbf{v}_{p} \in H\left(\operatorname{div} ; \Omega_{p}\right): \mathbf{v}_{p} \cdot \mathbf{n}_{p}=0 \text { on } \Gamma_{p}\right\}, \\
& Q_{p}:=L^{2}\left(\Omega_{p}\right) .
\end{aligned}
$$

For the spaces $\mathbf{X}_{f}, \mathbf{X}_{p}$, we define the following norms:

$$
\begin{aligned}
& \left\|\mathbf{v}_{f}\right\|_{1}=\sqrt{\left\|\mathbf{v}_{f}\right\|^{2}+\left|\mathbf{v}_{f}\right|_{1}^{2}}, \quad \text { with }\left|\mathbf{v}_{f}\right|_{1}=\left\|\nabla \mathbf{v}_{f}\right\| \forall \mathbf{v}_{f} \in \mathbf{X}_{f} \\
& \left\|\mathbf{v}_{p}\right\|_{\text {div }}=\sqrt{\left\|\mathbf{v}_{p}\right\|^{2}+\left\|\nabla \cdot \mathbf{v}_{p}\right\|^{2}} \quad \forall \mathbf{v}_{p} \in \mathbf{X}_{p} .
\end{aligned}
$$

The variational formulation of the steady-state Stokes-Darcy problem (2.1)-(2.7) reads as: Find $\left(\mathbf{u}_{f}, p ; \mathbf{u}_{p}, \phi\right) \in\left(\mathbf{X}_{f}, Q_{f} ; \mathbf{X}_{p}, Q_{p}\right)$ satisfying

$$
\begin{aligned}
& a_{f}\left(\mathbf{u}_{f}, \mathbf{v}_{f}\right)-b_{f}\left(\mathbf{v}_{f}, p\right)+c_{\Gamma}\left(\mathbf{v}_{f}, \phi\right)=\left(\mathbf{f}_{f}, \mathbf{v}_{f}\right) \quad \forall \mathbf{v}_{f} \in \mathbf{X}_{f}, \\
& b_{f}\left(\mathbf{u}_{f}, q\right)=0 \quad \forall q \in Q_{f}, \\
& a_{p}\left(\mathbf{u}_{p}, \mathbf{v}_{p}\right)-b_{p}\left(\mathbf{v}_{p}, \phi\right)-c_{\Gamma}\left(\mathbf{v}_{p}, \phi\right)=0 \quad \forall \mathbf{v}_{p} \in \mathbf{X}_{p}, \\
& b_{p}\left(\mathbf{u}_{p}, \psi\right)=\rho g\left(f_{p}, \psi\right) \quad \forall \psi \in Q_{p},
\end{aligned}
$$

where the bilinear forms are defined as

$$
\begin{aligned}
& a_{f}\left(\mathbf{u}_{f}, \mathbf{v}_{f}\right):=2 v\left(\mathbb{D}\left(\mathbf{u}_{f}\right), \mathbb{D}\left(\mathbf{v}_{f}\right)\right)+\sum_{i=1}^{d-1} \frac{\alpha}{\sqrt{\tau_{i} \cdot \mathbf{K} \tau_{i}}}\left(\mathbf{u}_{f} \cdot \tau_{i}, \mathbf{v}_{f} \cdot \tau_{i}\right)_{\Gamma}, \\
& a_{p}\left(\mathbf{u}_{p}, \mathbf{v}_{p}\right):=\rho g\left(\mathbf{K}^{-1} \mathbf{u}_{p}, \mathbf{v}_{p}\right) \\
& b_{f}\left(\mathbf{v}_{f}, p\right):=\left(p, \nabla \cdot \mathbf{v}_{f}\right) \\
& b_{p}\left(\mathbf{v}_{p}, \phi\right):=\rho g\left(\phi, \nabla \cdot \mathbf{v}_{p}\right) \\
& c_{\Gamma}\left(\mathbf{v}_{f}, \phi\right):=\rho g\left(\phi, \mathbf{v}_{f} \cdot \mathbf{n}_{f}\right)_{\Gamma} .
\end{aligned}
$$


After introducing

$$
\begin{gathered}
\mathcal{L}\left(\mathbf{u}_{f}, p, \mathbf{u}_{p}, \phi ; \mathbf{v}_{f}, q, \mathbf{v}_{p}, \psi\right):=a_{f}\left(\mathbf{u}_{f}, \mathbf{v}_{f}\right)-b_{f}\left(\mathbf{v}_{f}, p\right)+b_{f}\left(\mathbf{u}_{f}, q\right) \\
+a_{p}\left(\mathbf{u}_{p}, \mathbf{v}_{p}\right)-b_{p}\left(\mathbf{v}_{p}, \phi\right)+b_{p}\left(\mathbf{u}_{p}, \psi\right)+c_{\Gamma}\left(\mathbf{v}_{f}-\mathbf{v}_{p}, \phi\right)
\end{gathered}
$$

the variational formulation (3.1)-(3.4) can be equivalently rewritten as follows: Find $\left(\mathbf{u}_{f}, p ; \mathbf{u}_{p}, \phi\right) \in\left(\mathbf{X}_{f}, Q_{f} ; \mathbf{X}_{p}, Q_{p}\right)$ satisfying

$$
\mathcal{L}\left(\mathbf{u}_{f}, p, \mathbf{u}_{p}, \phi ; \mathbf{v}_{f}, q, \mathbf{v}_{p}, \psi\right)=\left(\mathbf{f}_{f}, \mathbf{v}_{f}\right)+\rho g\left(f_{p}, \psi\right)
$$

for all $\left(\mathbf{v}_{f}, q ; \mathbf{v}_{p}, \psi\right) \in\left(\mathbf{X}_{f}, Q_{f} ; \mathbf{X}_{p}, Q_{p}\right)$. It is easy to verify that this variational formulation is well-defined.

To end this section, we recall the following Poincaré, Korn's and the trace inequalities, which will be used in the later analysis. There exist constants $C_{P}, C_{K}, C_{\mathrm{v}}$, only depending on $\Omega_{f}$, such that for all $\mathbf{v}_{f} \in \mathbf{X}_{f}$,

$$
\left\|\mathbf{v}_{f}\right\| \leq C_{P}\left|\mathbf{v}_{f}\right|_{1}, \quad\left|\mathbf{v}_{f}\right|_{1} \leq C_{K}\left\|\mathbb{D}\left(\mathbf{v}_{f}\right)\right\|, \quad\left\|\mathbf{v}_{f}\right\|_{L^{2}(\Gamma)} \leq C_{\mathrm{v}}\left\|\mathbf{v}_{f}\right\|^{1 / 2}\left|\mathbf{v}_{f}\right|_{1}^{1 / 2} .
$$

Besides, there exists a constant $\tilde{C}_{\mathrm{v}}$ that only depends on $\Omega_{p}$ such that for all $\psi \in Q_{p}$,

$$
\|\psi\|_{L^{2}(\Gamma)} \leq \tilde{C}_{\mathrm{v}}\|\psi\|^{1 / 2}|\psi|_{1}^{1 / 2}
$$

Hereafer, all the constants are positive unless otherwise specified.

\section{The stabilized finite element method and its stability}

First, we consider the family of triangulations $T_{h}$ of $\Omega$, consisting of $T_{h}^{f}$ and $T_{h}^{p}$, which are regular triangulations of $\Omega_{f}$ and $\Omega_{p}$, respectively, where $h>0$ is a positive parameter. We also assume that on the interface $\Gamma$ the two meshes of $T_{h}^{f}$ and $T_{h}^{p}$, which form the regular triangulation $T_{h}:=T_{h}^{f} \cup T_{h}^{p}$, coincide.

The domain of the uniformly regular triangulation $\bar{\Omega}_{f} \cup \bar{\Omega}_{p}$ is such that $\bar{\Omega}=\{\cup K: K \in$ $\left.T_{h}\right\}$ and $h=\max _{K \in T_{h}} h_{K}$. There exist positive constants $c_{1}$ and $c_{2}$ satisfying $c_{1} h \leq h_{K} \leq$ $c_{2} \rho_{K}$. To approximate the diameter $h_{K}$ of the triangle (tetrahedral) $K, \rho_{K}$ is the diameter of the greatest ball included in $K$. Based on the subdivisions $T_{h}^{f}$ and $T_{h}^{p}$, we can define finite element spaces $\mathbf{X}_{f h} \subset \mathbf{X}_{f}, Q_{f h}^{h} \subset Q_{f}, \mathbf{X}_{p h} \subset \mathbf{X}_{p}, Q_{p h} \subset Q_{p}$. We consider the well-known MINI elements $(P 1 b-P 1)$ to approximate the velocity and pressure in the conduit for Stokes equation [68]. To capture the fully mixed technique in the porous medium region linear Lagrangian elements, $P 1$ are used for hydraulic (piezometric) head and Brezzi-DouglasMarini (BDM1) piecewise constant finite elements are used for Darcy velocity [69]. In the fluid flow region, we select for the Stokes problem the finite element spaces $\left(\mathbf{X}_{f h}, Q_{f h}\right)$ that satisfy the velocity-pressure inf-sup condition:

There exists a constant $\beta_{f}>0$, independent of h, such that

$$
\begin{aligned}
& \mathbf{X}_{f h} \subset \mathbf{X}_{f}, \quad Q_{f h} \subset Q_{f} \text {, }
\end{aligned}
$$

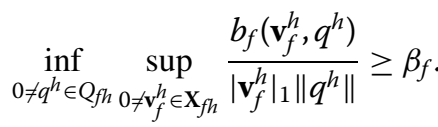


In the porous region, we use the finite element spaces $\left(\mathbf{X}_{p h}, Q_{p h}\right)$ that also satisfy a standard inf-sup condition:

There exists a $\beta_{p}>0$ such that for all $\phi^{h} \in Q_{p h}$,

$$
\begin{aligned}
& \mathbf{X}_{p h} \subset \mathbf{X}_{p}, \quad Q_{p h} \subset Q_{p}, \\
& \beta_{p}\left\|\phi^{h}\right\| \leq \sup _{\substack{0 \neq \mathbf{v}_{p}^{h} \in \mathbf{X}_{p h}\\
}} \frac{b_{p}\left(\mathbf{v}_{p}^{h}, \phi^{h}\right)}{\left\|\mathbf{v}_{p}^{h}\right\|_{\text {div }}} .
\end{aligned}
$$

From the inf-sup assumption (4.1), for a given arbitrary but fixed pressure $p^{h} \in Q_{f h}$, we can get $\mathbf{w}_{f}^{h} \in \mathbf{X}_{f h}$ such that

$$
b_{f}\left(\mathbf{w}_{f}^{h}, p^{h}\right) \geq \widetilde{C}_{1}\left\|\mathbf{w}_{f}^{h}\right\|_{1}\left\|p^{h}\right\| .
$$

By normalizing $\left\|\mathbf{w}_{f}^{h}\right\|_{1}=\lambda_{1}\left\|p^{h}\right\|$, thus

$$
b_{f}\left(\mathbf{w}_{f}^{h}, p^{h}\right) \geq C_{1}\left\|p^{h}\right\|^{2}
$$

In a similar way, from the inf-sup assumption (4.2), for $\phi^{h} \in Q_{p h}$, there exists a $\mathbf{w}_{p}^{h} \in \mathbf{X}_{p h}$ such that

$$
b_{p}\left(\mathbf{w}_{p}^{h}, \phi^{h}\right) \geq \widetilde{C}_{2}\left\|\mathbf{w}_{p}^{h}\right\|_{\text {div }}\left\|\phi^{h}\right\| .
$$

Assume that $\mathbf{w}_{p}^{h}$ is normalized so that $\left\|\mathbf{w}_{p}^{h}\right\|_{\text {div }}=\lambda_{2}\left\|\phi^{h}\right\|$, thus

$$
b_{p}\left(\mathbf{w}_{p}^{h}, \phi^{h}\right) \geq C_{2}\left\|\phi^{h}\right\|^{2} .
$$

Moreover, we need the inverse inequalities in both $\mathbf{X}_{f h}$ and $Q_{p h}$ : there exist constants $C_{\text {inv }}$ and $\tilde{C}_{\text {inv }}$, which depend on the minimum angles in the finite element mesh used on $\Omega_{f}$ and $\Omega_{p}$, such that

$$
\begin{aligned}
& \left|\mathbf{v}_{f}^{h}\right|_{1} \leq C_{\mathrm{inv}} h^{-1}\left\|\mathbf{v}_{f}^{h}\right\| \quad \forall \mathbf{v}_{f}^{h} \in \mathbf{X}_{f h}, \\
& \left|\psi^{h}\right|_{1} \leq \tilde{C}_{\mathrm{inv}} h^{-1}\left\|\psi^{h}\right\| \quad \forall \psi^{h} \in Q_{p h} .
\end{aligned}
$$

\subsection{The stabilized finite element method}

In this section, we present a stabilized finite element scheme for the Stokes-Darcy problem.

Algorithm 4.1 Find $\left(\mathbf{u}_{f}^{h}, p^{h}, \mathbf{u}_{p}^{h}, \phi^{h}\right) \in\left(\mathbf{X}_{f h}, Q_{f h}, \mathbf{X}_{p h}, Q_{p h}\right)$ satisfying

$$
\widetilde{\mathscr{L}}\left(\mathbf{u}_{f}^{h}, p^{h}, \mathbf{u}_{p}^{h}, \phi^{h} ; \mathbf{v}_{f}^{h}, q^{h}, \mathbf{v}_{p}^{h}, \psi^{h}\right)=\left(\mathbf{f}_{f}, \mathbf{v}_{f}^{h}\right)+\rho g\left(f_{p}, \psi^{h}\right),
$$

for any $\left(\mathbf{v}_{f}^{h}, q^{h}, \mathbf{v}_{p}^{h}, \psi^{h}\right) \in\left(\mathbf{X}_{f h}, Q_{f h}, \mathbf{X}_{p h}, Q_{p h}\right)$, where

$$
\begin{aligned}
\widetilde{\mathcal{L}}\left(\mathbf{u}_{f}^{h}, p^{h}, \mathbf{u}_{p}^{h}, \phi^{h} ; \mathbf{v}_{f}^{h}, q^{h}, \mathbf{v}_{p}^{h}, \psi^{h}\right)= & \mathcal{L}\left(\mathbf{u}_{f}^{h}, p^{h}, \mathbf{u}_{p}^{h}, \phi^{h} ; \mathbf{v}_{f}^{h}, q^{h} \mathbf{v}_{p}^{h}, \psi^{h}\right) \\
& +\frac{\delta}{h}\left(\left(\mathbf{u}_{f}^{h}-\mathbf{u}_{p}^{h}\right) \cdot \mathbf{n}_{f},\left(\mathbf{v}_{f}^{h}-\mathbf{v}_{p}^{h}\right) \cdot \mathbf{n}_{f}\right)_{\Gamma},
\end{aligned}
$$


and the term

$$
\frac{\delta}{h}\left(\left(\mathbf{u}_{f}^{h}-\mathbf{u}_{p}^{h}\right) \cdot \mathbf{n}_{f},\left(\mathbf{v}_{f}^{h}-\mathbf{v}_{p}^{h}\right) \cdot \mathbf{n}_{f}\right)_{\Gamma}=\frac{\delta}{h} \int_{\Gamma}\left(\left(\mathbf{u}_{f}^{h}-\mathbf{u}_{p}^{h}\right) \cdot \mathbf{n}_{f}\right)\left(\left(\mathbf{v}_{f}^{h}-\mathbf{v}_{p}^{h}\right) \cdot \mathbf{n}_{f}\right) d \Gamma,
$$

is the stabilized term for the Stokes-Darcy problem.

\subsection{The stability of the stabilized finite element method}

In this section, we prove the stability of the stabilized finite element scheme.

Theorem 1 (Continuity of $\widetilde{\mathcal{L}}$ ) There exists a constant $C$ such that

$$
\widetilde{\mathcal{L}}\left(\mathbf{u}_{f}^{h}, p^{h}, \mathbf{u}_{p}^{h}, \phi^{h} ; \mathbf{v}_{f}^{h}, q^{h}, \mathbf{v}_{p}^{h}, \psi^{h}\right) \leq C\left(\|\|\left(\mathbf{u}_{f}^{h}, p^{h}, \mathbf{u}_{p}^{h}, \phi^{h}\right)\|\|\left(\left\|\left(\mathbf{v}_{f}^{h}, q^{h}, \mathbf{v}_{p}^{h}, \psi^{h}\right)\right\|\right),\right.
$$

where

$$
\left\|\left(\mathbf{v}_{f}^{h}, q^{h}, \mathbf{v}_{p}^{h}, \psi^{h}\right)\right\|\|=\| \mathbf{v}_{f}^{h}\left\|_{1}+\right\| q^{h}\|+\| \mathbf{v}_{p}^{h}\left\|_{\text {div }}+\right\| \psi^{h}\left\|+h^{-1 / 2}\right\|\left(\mathbf{v}_{f}^{h}-\mathbf{v}_{p}^{h}\right) \|_{\Gamma} .
$$

Proof By the Schwarz inequality (3.6) and the inverse inequality (4.8), we have

$$
\begin{aligned}
c_{\Gamma}\left(\mathbf{u}_{f}^{h}-\mathbf{u}_{p}^{h}, \phi^{h}\right) & \leq \rho g\left\|\left(\mathbf{u}_{f}^{h}-\mathbf{u}_{p}^{h}\right) \cdot \mathbf{n}_{f}\right\|_{\Gamma}\left\|\phi^{h}\right\|_{\Gamma} \\
& \leq \rho g \tilde{C}_{\mathrm{v}} \tilde{C}_{\mathrm{inv}} h^{-1 / 2}\left\|\left(\mathbf{u}_{f}^{h}-\mathbf{u}_{p}^{h}\right) \cdot \mathbf{n}_{f}\right\|_{\Gamma}\left\|\phi^{h}\right\|
\end{aligned}
$$

and

$$
\begin{aligned}
& \frac{\delta}{h}\left(\left(\mathbf{u}_{f}^{h}-\mathbf{u}_{p}^{h}\right) \cdot \mathbf{n}_{f},\left(\mathbf{v}_{f}^{h}-\mathbf{v}_{p}^{h}\right) \cdot \mathbf{n}_{f}\right)_{\Gamma} \\
& \quad \leq \delta h^{-1 / 2}\left\|\left(\mathbf{u}_{f}^{h}-\mathbf{u}_{p}^{h}\right) \cdot \mathbf{n}_{f}\right\|_{\Gamma} h^{-1 / 2}\left\|\left(\mathbf{v}_{f}^{h}-\mathbf{v}_{p}^{h}\right) \cdot \mathbf{n}_{f}\right\|_{\Gamma} .
\end{aligned}
$$

Thus we can prove that $\widetilde{\mathscr{L}}$ is continuous, i.e.,

$$
\begin{aligned}
& \widetilde{\mathcal{L}}\left(\mathbf{u}_{f}^{h}, p^{h}, \mathbf{u}_{p}^{h}, \phi^{h} ; \mathbf{v}_{f}^{h}, q^{h}, \mathbf{v}_{p}^{h}, \psi^{h}\right) \\
&=a_{f}\left(\mathbf{u}_{f}^{h}, \mathbf{v}_{f}^{h}\right)-b_{f}\left(\mathbf{v}_{f}^{h}, p^{h}\right)+b_{f}\left(\mathbf{u}_{f}^{h}, q^{h}\right) \\
& \quad+a_{p}\left(\mathbf{u}_{p}^{h}, \mathbf{v}_{p}^{h}\right)-b_{p}\left(\mathbf{v}_{p}^{h}, \phi^{h}\right)+b_{p}\left(\mathbf{u}_{p}^{h}, \psi^{h}\right) \\
& \quad+\frac{\delta}{h}\left(\left(\mathbf{u}_{f}^{h}-\mathbf{u}_{p}^{h}\right) \cdot \mathbf{n}_{f},\left(\mathbf{v}_{f}^{h}-\mathbf{v}_{p}^{h}\right) \cdot \mathbf{n}_{f}\right)+c_{\Gamma}\left(\mathbf{v}_{f}^{h}-\mathbf{u}_{p}^{h}, \phi^{h}\right) \\
& \leq C\left(\left\|\left(\mathbf{u}_{f}^{h}, p^{h}, \mathbf{u}_{p}^{h}, \phi^{h}\right)\right\|\right)\left(\left\|\left(\mathbf{v}_{f}^{h}, q^{h}, \mathbf{v}_{p}^{h}, \psi^{h}\right)\right\|\right) .
\end{aligned}
$$

The proof is complete.

Theorem 2 (Coercivity of $\widetilde{\mathcal{L}}$ ) There exists a constant $\beta>0$ such that the following inequality holds for all $\left(\mathbf{u}_{f}^{h}, p^{h}, \mathbf{u}_{p}^{h}, \phi^{h}\right) \in\left(\mathbf{X}_{f h}, Q_{f h}, \mathbf{X}_{p h}, Q_{p h}\right)$ :

$$
\sup _{\left(\mathbf{v}_{f}^{h}, q^{h}, \mathbf{v}_{p}^{h}, \psi^{h}\right) \in\left(X_{f h}, Q_{f h}, X_{p h}, Q_{p h}\right)} \frac{\widetilde{\mathcal{L}}\left(\mathbf{u}_{f}^{h}, p^{h}, \mathbf{u}_{p}^{h}, \phi^{h} ; \mathbf{v}_{f}^{h}, q^{h}, \mathbf{v}_{p}^{h}, \psi^{h}\right)}{\left\|\left(\mathbf{v}_{f}^{h}, q^{h}, \mathbf{v}_{p}^{h}, \psi^{h}\right)\right\|} \geq \beta\left\|\left(\mathbf{u}_{f}^{h}, p^{h}, \mathbf{u}_{p}^{h}, \phi^{h}\right)\right\| .
$$


Proof We will construct $\left(\widehat{\mathbf{v}_{f}^{h}}, \widehat{q^{h}}, \widehat{\mathbf{v}_{p}^{h}}, \widehat{\psi^{h}}\right)$ such that

$$
\widetilde{\mathcal{L}}\left(\mathbf{u}_{f}^{h}, p^{h}, \mathbf{u}_{p}^{h}, \phi^{h} ; \widehat{\mathbf{v}_{f}^{h}}, \widehat{q^{h}}, \widehat{\mathbf{v}_{p}^{h}}, \widehat{\psi^{h}}\right) \geq C\left(\left\|\left(\mathbf{u}_{f}^{h}, p^{h}, \mathbf{u}_{p}^{h}, \phi^{h}\right)\right\|\right)\left(\left\|\left(\widehat{\mathbf{v}_{f}^{h}}, \widehat{q^{h}}, \widehat{\mathbf{v}_{p}^{h}}, \widehat{\psi^{h}}\right)\right\|\right)
$$

using the following three steps.

Step 1. By setting $\left(\mathbf{v}_{f}^{h}, q^{h}, \mathbf{v}_{p}^{h}, \psi^{h}\right)=\left(\mathbf{u}_{f}^{h}, p^{h}, \mathbf{u}_{p}^{h}, \phi^{h}+\nabla \cdot \mathbf{u}_{p}^{h}\right)$, we derive

$$
\begin{aligned}
& \widetilde{\mathscr{L}}\left(\mathbf{u}_{f}^{h}, p^{h}, \mathbf{u}_{p}^{h}, \phi^{h} ; \mathbf{u}_{f}^{h}, p^{h}, \mathbf{u}_{p}^{h}, \phi^{h}+\nabla \cdot \mathbf{u}_{p}^{h}\right) \\
& \quad=\left\|\mathbf{u}_{f}^{h}\right\|_{1}^{2}+\left\|\mathbf{u}_{p}^{h}\right\|_{\text {div }}^{2}+\frac{\delta}{h}\left\|\left(\mathbf{u}_{f}^{h}-\mathbf{u}_{p}^{h}\right) \cdot \mathbf{n}_{f}\right\|_{\Gamma}^{2}+c_{\Gamma}\left(\mathbf{u}_{f}^{h}-\mathbf{u}_{p}^{h}, \phi^{h}\right) .
\end{aligned}
$$

Note that

$$
\begin{aligned}
c_{\Gamma}\left(\mathbf{u}_{f}^{h}-\mathbf{u}_{p}^{h}, \phi^{h}\right) & \geq-\rho g \tilde{C}_{\mathrm{v}} \tilde{C}_{\mathrm{inv}} h^{-1 / 2}\left\|\left(\mathbf{u}_{f}^{h}-\mathbf{u}_{p}^{h}\right) \cdot \mathbf{n}_{f}\right\|_{\Gamma}\left\|\phi^{h}\right\| \\
& \geq-\frac{\rho^{2} g^{2} \tilde{C}_{\mathrm{v}}^{2} \tilde{C}_{\mathrm{inv}}^{2}}{\gamma h C_{2}}\left\|\left(\mathbf{u}_{f}^{h}-\mathbf{u}_{p}^{h}\right) \cdot \mathbf{n}_{f}\right\|_{\Gamma}^{2}-\frac{\gamma C_{2}}{4}\left\|\phi^{h}\right\|^{2},
\end{aligned}
$$

where $\gamma$ is a real positive parameter, which will be determined later.

Step 2. By selecting $\left(\mathbf{v}_{f}^{h}, q^{h}, \mathbf{v}_{p}^{h}, \psi^{h}\right)=\left(-\gamma \mathbf{w}_{f}^{h}, 0,-\gamma \mathbf{w}_{p}^{h}, 0\right)$ where $\mathbf{w}_{f}^{h}, \mathbf{w}_{p}^{h}$ satisfy (4.5) and (4.6), respectively, and normalizing $\left\|\left(\mathbf{w}_{f}^{h}-\mathbf{w}_{p}^{h}\right) \cdot \mathbf{n}_{f}\right\|_{\Gamma}=\lambda_{3}\left\|\left(\mathbf{u}_{f}^{h}-\mathbf{u}_{p}^{h}\right) \cdot \mathbf{n}_{f}\right\|_{\Gamma}$, together with (4.3)-(4.6), we have

$$
\begin{aligned}
& \widetilde{L}\left(\mathbf{u}_{f}^{h}, p^{h}, \mathbf{u}_{p}^{h}, \phi^{h} ;-\gamma \mathbf{w}_{f}^{h}, 0,-\gamma \mathbf{w}_{p}^{h}, 0\right) \\
&=-\gamma a_{f}\left(\mathbf{u}_{f}^{h}, \mathbf{w}_{f}^{h}\right)-\gamma a_{p}\left(\mathbf{u}_{p}^{h}, \mathbf{w}_{p}^{h}\right)+\gamma b_{f}\left(\mathbf{w}_{f}^{h}, p^{h}\right)+\gamma b_{p}\left(\mathbf{w}_{p}^{h}, \phi^{h}\right) \\
& \quad-\frac{\gamma \delta}{h}\left(\left(\mathbf{u}_{f}^{h}-\mathbf{u}_{p}^{h}\right) \cdot \mathbf{n}_{f},\left(\mathbf{w}_{f}^{h}-\mathbf{w}_{p}^{h}\right) \cdot \mathbf{n}_{f}\right)_{\Gamma}-\gamma c_{\Gamma}\left(\mathbf{w}_{f}^{h}-\mathbf{w}_{p}^{h}, \phi^{h}\right) \\
& \geq-\gamma \lambda_{1}\left\|\mathbf{u}_{f}^{h}\right\|_{1}\left\|p^{h}\right\|-\gamma \lambda_{2}\left\|\mathbf{u}_{p}^{h}\right\|_{\text {div }}\left\|\phi^{h}\right\|+\gamma C_{1}\left\|p^{h}\right\|^{2}+\gamma C_{2}\left\|\phi^{h}\right\|^{2} \\
&-\frac{\gamma \delta \lambda_{3}}{h}\left\|\left(\mathbf{u}_{f}^{h}-\mathbf{u}_{p}^{h}\right) \cdot \mathbf{n}_{f}\right\|_{\Gamma}^{2}-\gamma \lambda_{3} \rho g \tilde{C}_{\mathrm{v}} \tilde{C}_{\mathrm{inv}} h^{-1 / 2}\left\|\left(\mathbf{u}_{f}^{h}-\mathbf{u}_{p}^{h}\right) \cdot \mathbf{n}_{f}\right\|_{\Gamma}\left\|\phi^{h}\right\| \\
& \geq-\frac{\gamma \lambda_{1}^{2}}{2 C_{1}}\left\|\mathbf{u}_{f}^{h}\right\|_{1}^{2}-\frac{\gamma \lambda_{2}^{2}}{C_{2}}\left\|\mathbf{u}_{p}^{h}\right\|_{\text {div }}^{2}+\frac{\gamma C_{1}}{2}\left\|p^{h}\right\|^{2}+\frac{\gamma C_{2}}{2}\left\|\phi^{h}\right\|^{2} \\
&-\frac{\gamma \delta \lambda_{3}}{h}\left\|\left(\mathbf{u}_{f}^{h}-\mathbf{u}_{p}^{h}\right) \cdot \mathbf{n}_{f}\right\|_{\Gamma}^{2}-\frac{\gamma \lambda_{3}^{2} \rho^{2} g^{2} \tilde{C}_{\mathrm{v}}^{2} \tilde{C}_{\mathrm{inv}}^{2}}{h C_{2}}\left\|\left(\mathbf{u}_{f}^{h}-\mathbf{u}_{p}^{h}\right) \cdot \mathbf{n}_{f}\right\|_{\Gamma}^{2},
\end{aligned}
$$

here the following two main Young inequalities are used:

$$
\begin{aligned}
& \gamma \lambda_{2}\left\|\mathbf{u}_{p}^{h}\right\|_{\text {div }}\left\|\phi^{h}\right\| \leq \frac{\gamma \lambda_{2}^{2}}{C_{2}}\left\|\mathbf{u}_{p}^{h}\right\|_{\text {div }}^{2}+\frac{\gamma C_{2}}{4}\left\|\phi^{h}\right\|^{2}, \\
& \gamma \lambda_{3} \rho g \tilde{C}_{\mathrm{v}} \tilde{C}_{\mathrm{inv}} h^{-1 / 2}\left\|\left(\mathbf{u}_{f}^{h}-\mathbf{u}_{p}^{h}\right) \cdot \mathbf{n}_{f}\right\|_{\Gamma}\left\|\phi^{h}\right\| \\
& \quad \leq \frac{\gamma \lambda_{3}^{2} \rho^{2} g^{2} \tilde{C}_{\mathrm{v}}^{2} \tilde{C}_{\mathrm{inv}}^{2}}{h C_{2}}\left\|\left(\mathbf{u}_{f}^{h}-\mathbf{u}_{p}^{h}\right) \cdot \mathbf{n}_{f}\right\|_{\Gamma}^{2}+\frac{\gamma C_{2}}{4}\left\|\phi^{h}\right\|^{2},
\end{aligned}
$$

where $\delta$ is another real positive parameter to be determined soon. 
Step 3. Choosing $\left(\widehat{\mathbf{v}_{f}^{h}}, \widehat{q^{h}}, \widehat{\mathbf{v}_{p}^{h}}, \widehat{\psi^{h}}\right)=\left(\mathbf{u}_{f}^{h}-\gamma \mathbf{w}_{f}^{h}, p^{h}, \mathbf{u}_{p}^{h}-\gamma \mathbf{w}_{p}^{h}, \phi^{h}+\nabla \cdot \mathbf{u}_{p}^{h}\right)$, we can obtain by the above arguments the following inequality:

$$
\begin{aligned}
& \tilde{\mathscr{L}}\left(\mathbf{u}_{f}^{h}, p^{h}, \mathbf{u}_{p}^{h}, \phi^{h} ; \mathbf{u}_{f}^{h}-\gamma \mathbf{w}_{f}^{h}, p^{h}, \mathbf{u}_{p}^{h}-\gamma \mathbf{w}_{p}^{h}, \phi^{h}+\nabla \cdot \mathbf{u}_{p}^{h}\right) \\
& \geq\left(1-\frac{\gamma \lambda_{1}^{2}}{2 C_{1}}\right)\left\|\mathbf{u}_{f}\right\|^{2}+\left(1-\frac{\gamma \lambda_{2}^{2}}{C_{2}}\right)\left\|\mathbf{u}_{p}^{h}\right\|_{\text {div }}^{2}+\frac{\gamma C_{1}}{2}\left\|p^{h}\right\|^{2}+\frac{\gamma C_{2}}{4}\left\|\phi^{h}\right\|^{2} \\
& \quad+\left(\frac{\delta\left(1-\gamma \lambda_{3}\right)}{h}-\frac{\rho^{2} g^{2} \tilde{C}_{\mathrm{v}}^{2} \tilde{C}_{\text {inv }}^{2}}{\gamma h C_{2}}-\frac{\gamma \lambda_{3}^{2} \rho^{2} g^{2} \tilde{C}_{\mathrm{v}}^{2} \tilde{C}_{\mathrm{inv}}^{2}}{h C_{2}}\right)\left\|\left(\mathbf{u}_{f}^{h}, p^{h}, \mathbf{u}_{p}^{h}, \phi^{h}\right)\right\|^{2} .
\end{aligned}
$$

Then we enforce the following conditions on $\gamma$ and $\delta$ :

$$
\begin{aligned}
& 1-\frac{\gamma \lambda_{1}^{2}}{2 C_{1}} \geq \frac{1}{2}, \quad 1-\frac{\gamma \lambda_{2}^{2}}{C_{2}} \geq \frac{1}{2}, \quad 1-\gamma \lambda_{3} \geq \frac{1}{4}, \\
& \frac{\delta\left(1-\gamma \lambda_{3}\right)}{h}-\frac{\rho^{2} g^{2} \tilde{C}_{\mathrm{v}}^{2} \tilde{C}_{\mathrm{inv}}^{2}}{\gamma h C_{2}}-\frac{\gamma \lambda_{3}^{2} \rho^{2} g^{2} \tilde{C}_{\mathrm{v}}^{2} \tilde{C}_{\mathrm{inv}}^{2}}{h C_{2}} \geq \frac{\delta}{2 h} .
\end{aligned}
$$

This encourages us to select sufficiently small $\gamma$ and large $\delta$ as follows:

$$
\gamma \leq \min \left\{\frac{C_{1}}{\lambda_{1}^{2}}, \frac{2 C_{2}}{\lambda_{2}^{2}}, \frac{1}{4 \lambda_{3}}\right\}, \quad \delta \geq \frac{4 \rho^{2} g^{2} \tilde{C}_{\mathrm{v}}^{2} \tilde{C}_{\mathrm{inv}}^{2}\left(1+\gamma^{2} \lambda_{3}^{2}\right)}{\gamma C_{2}} .
$$

To this end, we can obtain

$$
\begin{aligned}
& \widetilde{\mathcal{L}}\left(\mathbf{u}_{f}^{h}, p^{h}, \mathbf{u}_{p}^{h}, \phi^{h} ; \mathbf{u}_{f}^{h}-\gamma \mathbf{w}_{f}^{h}, p^{h}, \mathbf{u}_{p}^{h}-\gamma \mathbf{w}_{p}^{h}, \phi^{h}+\nabla \cdot \mathbf{u}_{p}^{h}\right) \\
& \geq\left(1-\frac{\gamma \lambda_{1}^{2}}{2 C_{1}}\right)\left\|\mathbf{u}_{f}\right\|^{2}+\left(1-\frac{\gamma \lambda_{2}^{2}}{C_{2}}\right)\left\|\mathbf{u}_{p}^{h}\right\|_{\mathrm{div}}^{2}+\frac{\gamma C_{1}}{2}\left\|p^{h}\right\|^{2}+\frac{\gamma C_{2}}{4}\left\|\phi^{h}\right\|^{2} \\
&+\left(\frac{\delta}{h}-\frac{\gamma \delta \lambda_{3}}{h}-\frac{\rho^{2} g^{2} \tilde{C}_{\mathrm{v}}^{2} \tilde{C}_{\mathrm{inv}}^{2}}{\gamma h C_{2}}-\frac{\gamma \lambda_{3}^{2} \rho^{2} g^{2} \tilde{C}_{\mathrm{v}}^{2} \tilde{C}_{\mathrm{inv}}^{2}}{h C_{2}}\right)\left\|\left(\mathbf{u}_{f}^{h}, p^{h}, \mathbf{u}_{p}^{h}, \phi^{h}\right)\right\|^{2} \\
& \geq C_{4}\left(\left\|\left(\mathbf{u}_{f}^{h}, p^{h}, \mathbf{u}_{p}^{h}, \phi^{h}\right)\right\|\right)\left(\left\|\left(\mathbf{u}_{f}^{h}-\gamma \mathbf{w}_{f}^{h}, p^{h}, \mathbf{u}_{p}^{h}-\gamma \mathbf{w}_{p}^{h}, \phi^{h}+\nabla \cdot \mathbf{u}_{p}^{h}\right)\right\|\right) \\
&= C\left(\left\|\left(\mathbf{u}_{f}^{h}, p^{h}, \mathbf{u}_{p}^{h}, \phi^{h}\right)\right\|\right)\left(\left\|\left(\widehat{\mathbf{v}_{f}^{h}}, \widehat{q^{h}}, \widehat{\mathbf{v}_{p}^{h}}, \widehat{\psi^{h}}\right)\right\|\right) .
\end{aligned}
$$

The proof is complete.

\section{Error estimate for the stabilized finite element method}

In this section, we prove the error estimate for the stabilized finite element method for the scheme.

Theorem 3 Assume that $\left(\mathbf{u}_{f}, p ; \mathbf{u}_{p}, \phi\right)$ is an exact solution, the norms $\left\|\mathbf{u}_{p}\right\|_{2},\left\|\mathbf{u}_{f}\right\|_{2},\|p\|_{1}$, and $\|\phi\|_{1}$ are bounded from above, and, moreover, $\left(\mathbf{u}_{f}^{h}, p^{h} ; \mathbf{u}_{p}^{h}, \phi^{h}\right)$ is the stabilized finite element solution, then we have

$$
\left\|\mathbf{u}_{f}-\mathbf{u}_{f}^{h}\right\|_{1}+\left\|p-p^{h}\right\|+\left\|\mathbf{u}_{p}-\mathbf{u}_{p}^{h}\right\|_{\mathrm{div}}+\left\|\phi-\phi^{h}\right\| \leq C h .
$$


Proof First, by subtracting (4.9) from (3.5), thanks to the interface condition (2.5), we get the error equation as follows:

$$
\begin{aligned}
\mathscr{L} & \left(\mathbf{u}_{f}, p, \mathbf{u}_{p}, \phi ; \mathbf{v}_{f}^{h}, q^{h}, \mathbf{v}_{p}^{h}, \psi^{h}\right)-\widetilde{\mathcal{L}}\left(\mathbf{u}_{f}^{h}, p^{h}, \mathbf{u}_{p}^{h}, \phi^{h} ; \mathbf{v}_{f}^{h}, q^{h}, \mathbf{v}_{p}^{h}, \psi^{h}\right) \\
& =\widetilde{\mathcal{L}}\left(\mathbf{u}_{f}, p, \mathbf{u}_{p}, \phi ; \mathbf{v}_{f}^{h}, q^{h}, \mathbf{v}_{p}^{h}, \psi^{h}\right)-\widetilde{\mathcal{L}}\left(\mathbf{u}_{f}^{h}, p^{h}, \mathbf{u}_{p}^{h}, \phi^{h} ; \mathbf{v}_{f}^{h}, q^{h}, \mathbf{v}_{p}^{h}, \psi^{h}\right) \\
& =\widetilde{\mathcal{L}}\left(\mathbf{u}_{f}-\mathbf{u}_{f}^{h}, p-p^{h}, \mathbf{u}_{p}-\mathbf{u}_{p}^{h}, \phi-\phi^{h} ; \mathbf{v}_{f}^{h}, q^{h}, \mathbf{v}_{p}^{h}, \psi^{h}\right)=0 .
\end{aligned}
$$

By introducing $\left(\overline{\mathbf{u}}, \bar{p}, \overline{\mathbf{u}}_{p}, \bar{\phi}\right)$ as the interpolation of $\left(\mathbf{u}_{f}, p, \mathbf{u}_{p}, \phi\right)$ from $\left(\mathbf{X}_{f}, Q_{f}, \mathbf{X}_{p}, Q_{p}\right)$ onto the finite element spaces $\left(\mathbf{X}_{f h}, Q_{f h}, \mathbf{X}_{p h}, Q_{p h}\right)$, we split the errors into two parts as

$$
\begin{aligned}
& \mathbf{u}_{f}-\mathbf{u}_{f}^{h}=\left(\mathbf{u}_{f}-\overline{\mathbf{u}}_{f}\right)+\left(\overline{\mathbf{u}}_{f}-\mathbf{u}_{f}^{h}\right) \triangleq \overline{\mathbf{e}}_{f}+\mathbf{e}_{f}^{h}, \\
& p-p^{h}=(p-\bar{p})+\left(\bar{p}-p^{h}\right) \triangleq \bar{\eta}+\eta^{h}, \\
& \mathbf{u}_{p}-\mathbf{u}_{p}^{h}=\left(\mathbf{u}_{p}-\overline{\mathbf{u}}_{p}\right)+\left(\overline{\mathbf{u}}_{p}-\mathbf{u}_{p}^{h}\right) \triangleq \overline{\mathbf{e}}_{p}+\mathbf{e}_{p}^{h}, \\
& \phi-\phi^{h}=(\phi-\bar{\phi})+\left(\bar{\phi}-\phi^{h}\right) \triangleq \bar{\theta}+\theta^{h},
\end{aligned}
$$

The interpolation error are listed below:

$$
\left\|\overline{\mathbf{e}}_{f}\right\|_{1}+\|\bar{\eta}\| \leq C h\left(\left\|\mathbf{u}_{f}\right\|_{2}+\|p\|_{1}\right), \quad\left\|\overline{\mathbf{e}}_{p}\right\|_{\text {div }}+\|\bar{\theta}\| \leq C h\left(\left\|\mathbf{u}_{p}\right\|_{2}+\|\phi\|_{1}\right) .
$$

Substituting them into the error equation (5.2), we get

$$
\widetilde{\mathcal{L}}\left(\mathbf{e}_{f}^{h}, \eta^{h}, \mathbf{e}_{p}^{h}, \theta^{h} ; \mathbf{v}_{f}^{h}, q^{h}, \mathbf{v}_{p}^{h}, \psi^{h}\right)=-\widetilde{\mathscr{L}}\left(\overline{\mathbf{e}}_{f}, \bar{\eta}, \overline{\mathbf{e}}_{p}, \bar{\theta} ; \mathbf{v}_{f}^{h}, q^{h}, \mathbf{v}_{p}^{h}, \psi^{h}\right) .
$$

From the coercivity and the continuity of $\widetilde{\mathcal{L}}$, trace and inverse inequalities, we obtain

$$
\begin{aligned}
& \beta\left\|\left(\mathbf{e}_{f}^{h}, \eta^{h}, \mathbf{e}_{p}^{h}, \theta^{h}\right)\right\| \\
& \quad \leq \sup _{\left(\mathbf{v}_{f}^{h}, q^{h}, \mathbf{v}_{p}^{h}, \psi^{h}\right)} \frac{\widetilde{\mathscr{L}}\left(\mathbf{e}_{f}^{h}, \eta^{h}, \mathbf{e}_{p}^{h}, \theta^{h} ; \mathbf{v}_{f}^{h}, q^{h}, \mathbf{v}_{p}^{h}, \psi^{h}\right)}{\left\|\left(\mathbf{v}_{f}^{h}, q^{h}, \mathbf{v}_{p}^{h}, \psi^{h}\right)\right\|} \\
& \quad=\sup _{\left(\mathbf{v}_{f}^{h}, q^{h}, \mathbf{v}_{p}^{h}, \psi^{h}\right)} \frac{-\widetilde{\mathcal{L}}\left(\overline{\mathbf{e}}_{f}, \bar{\eta}, \overline{\mathbf{e}}_{p}, \bar{\theta} ; \mathbf{v}_{f}^{h}, q^{h}, \mathbf{v}_{p}^{h}, \psi^{h}\right)}{\left\|\left(\mathbf{v}_{f}^{h}, q^{h}, \mathbf{v}_{p}^{h}, \psi^{h}\right)\right\|} \\
& \leq C\left\|\left(\overline{\mathbf{e}}_{f}, \bar{\eta}, \overline{\mathbf{e}}_{p}, \bar{\theta}\right)\right\| \| C\left(\left\|\overline{\mathbf{e}}_{f}\right\|_{1}+\|\bar{\eta}\|+\left\|\overline{\mathbf{e}}_{p}\right\|_{\text {div }}+\|\bar{\theta}\|+h^{-1 / 2}\left\|\left(\overline{\mathbf{e}}_{f}-\overline{\mathbf{e}}_{p}\right)\right\|_{\Gamma}\right) \\
& \leq C\left(\left\|\overline{\mathbf{e}}_{f}\right\|_{1}+\|\bar{\eta}\|+\left\|\overline{\mathbf{e}}_{p}\right\|_{\text {div }}+\|\bar{\theta}\|+h^{-1}\left(\left\|\overline{\mathbf{e}}_{f}\right\|+\left\|\overline{\mathbf{e}}_{p}\right\|\right)\right) \\
& \leq C h\left(\left\|\mathbf{u}_{f}\right\|_{2}+\|p\|_{1}+\left\|\mathbf{u}_{p}\right\|_{2}+\|\phi\|_{1}\right) .
\end{aligned}
$$

Finally, together with the interpolation error, we derive the error estimate (5.2).

\section{Numerical experiments}

In this section, we present two numerical experiments to illustrate the accuracy and efficiency of the proposed stabilized fully mixed finite element method. The global domain $\Omega$ consists of two subdomains with free fluid flow region $\Omega_{f}=[0,1] \times[1,2]$ and porous 
medium domain $\Omega_{p}=[0,1] \times[0,1]$ for both numerical tests. The interface of the current computational domain is $\Gamma=[0,1] \times\{1\}$.

The finite element spaces are constructed by the well-known MINI elements $(P 1 b-P 1)$ for Stokes problem. To capture the fully mixed technique in the porous medium region, linear Lagrangian elements $P 1$ are used for hydraulic (piezometric) head and BrezziDouglas-Marini (BDM1) piecewise constant finite elements are used for Darcy velocity. In the first numerical test, the influence of the stabilization parameter on the temporal discretization is examined by considering different values of $\delta$ and then the optimal convergence for the spacing $h$ is checked. In the second numerical test, we focus on showing the effects of the hydraulic conductivity parameter $K$ for gradual decreasing. All the numerical tests are executed by a specialized free domain software known as FreeFEM++ [70]. Graphs and figures are drawn by using MATLAB and Tecplot software.

\subsection{Convergence test 1}

As the first numerical test example, we choose the following exact solution, which satisfies the Beavers-Joseph-Saffman interface conditions (2.5)-(2.7):

$$
\left\{\begin{array}{l}
\mathbf{u}_{f}=\left(\begin{array}{c}
{\left[x^{2}(y-1)^{2}+y\right]} \\
{\left[-\frac{2}{3} x(y-1)^{3}\right]+[2-\pi \sin (\pi x)]}
\end{array}\right), \\
p=[2-\pi \sin (\pi x)] \sin (0.5 \pi y), \\
\phi=[2-\pi \sin (\pi x)][1-y-\cos (\pi y)], \\
\mathbf{u}_{p}=-\mathbf{K} \nabla \phi .
\end{array}\right.
$$

The Dirichlet boundary condition and source term of the model problem are chosen in such a way that the above-listed functions are the exact solutions of the model problem. For computational convenience in the first numerical experiment, all the physical parameters $v, \rho, g, \mathbf{K}, \alpha$ are simply taken as 1.0.

To investigate the impact of the stabilization parameter on the convergence order for the finite element scheme, we perform several numerical tests from different aspects in the following. First, for the different values of $\delta=10^{k}(k=3,2,1,-1,-2,-3,-4,-5,0)$, we use Fig. 2 to demonstrate the order of the convergence of $\left\|\mathbf{u}_{f}^{h}-\mathbf{u}_{f}\right\|_{1, \Omega_{f}}$ and $\left\|\mathbf{u}_{p}^{h}-\mathbf{u}_{p}\right\|_{0, \Omega_{p}}$. This study admits that the relatively large and small values of the stabilization parameter $\delta$ significantly affect the convergence orders of velocity and/or pressure. Without assuming the stabilization parameter $\delta$ (namely, $\delta=0$ ), the convergence order is also not accurate. We can obtain the accurate convergence order for the value of the stabilization parameter $\delta=0.1$. In Figs. 3 and 4, we present the velocity and pressure contours for the approximate and exact solutions with the different values of the stabilization parameter $\delta=0.1,1.0,1000,0.00001$ and 0 . These figures also illustrate that the velocity and pressure contours are simultaneously regular for an approximate and exact solutions with the value of the stabilization parameter $\delta=0.1$. In fact, considering the small values of the stabilization parameter $\delta=0.0001$ and 0 , the velocity contour changed dramatically for approximate solutions. The same illustration can be seen for the large values of $\delta=1000$ on the pressure contour. From the figures, we can conclude that for the relatively small and large values of the stabilization parameter $\delta$ cause a significant effect on the accuracy of the convergence order, velocity and pressure contours. The above discussion leads us 

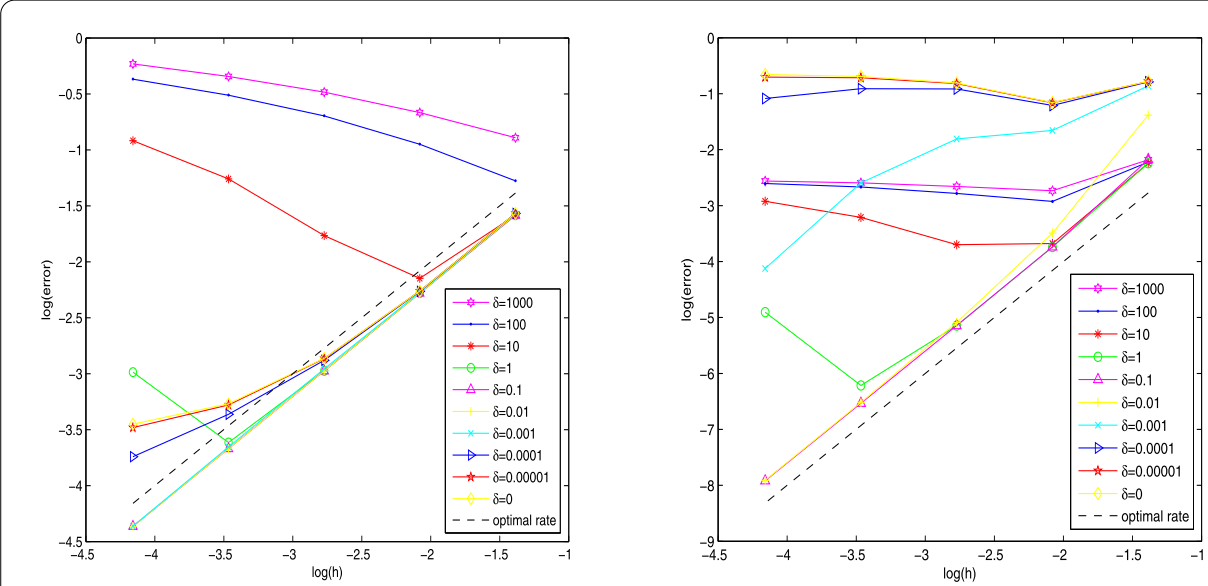

Figure 2 The order of convergence for the different values of the stabilization parameter $\delta$ for test 1. (Left) The order of the convergence of $\left\|\mathbf{u}_{f}^{h}-\mathbf{u}_{f}\right\|_{1, \Omega_{f}}$; (Right) The order of the convergence of $\left\|\mathbf{u}_{p}^{h}-\mathbf{u}_{p}\right\|_{0, \Omega_{p}}$
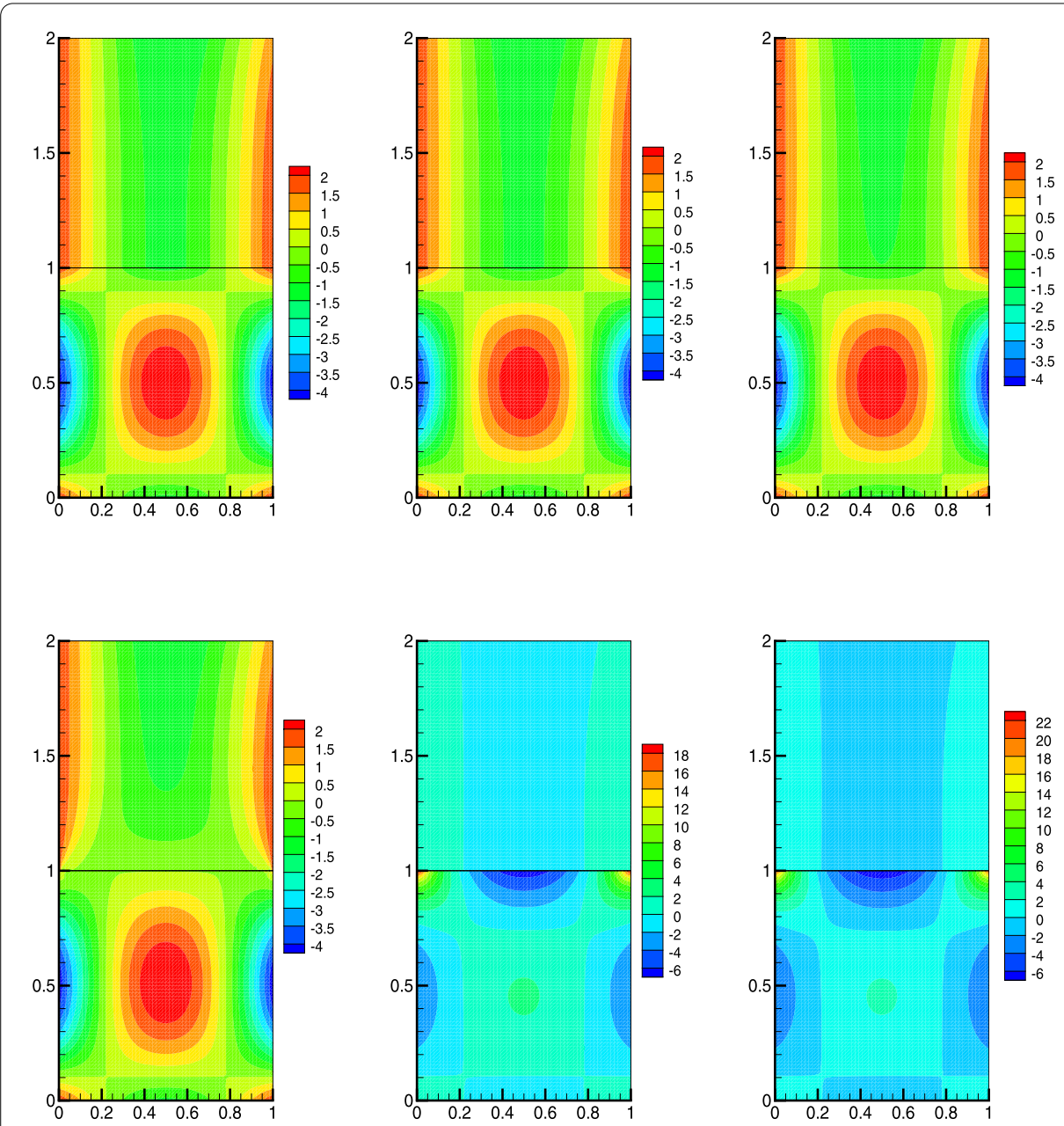

Figure 3 An illustration of the velocity contour with different values of the stabilization parameter for test 1: true solution (top-left); $\delta=0.1$ (top-middle); $\delta=1$ (top-right); $\delta=1000$ (down-left); $\delta=0.0001$ (down-middle); $\delta=0$ (down-right) 

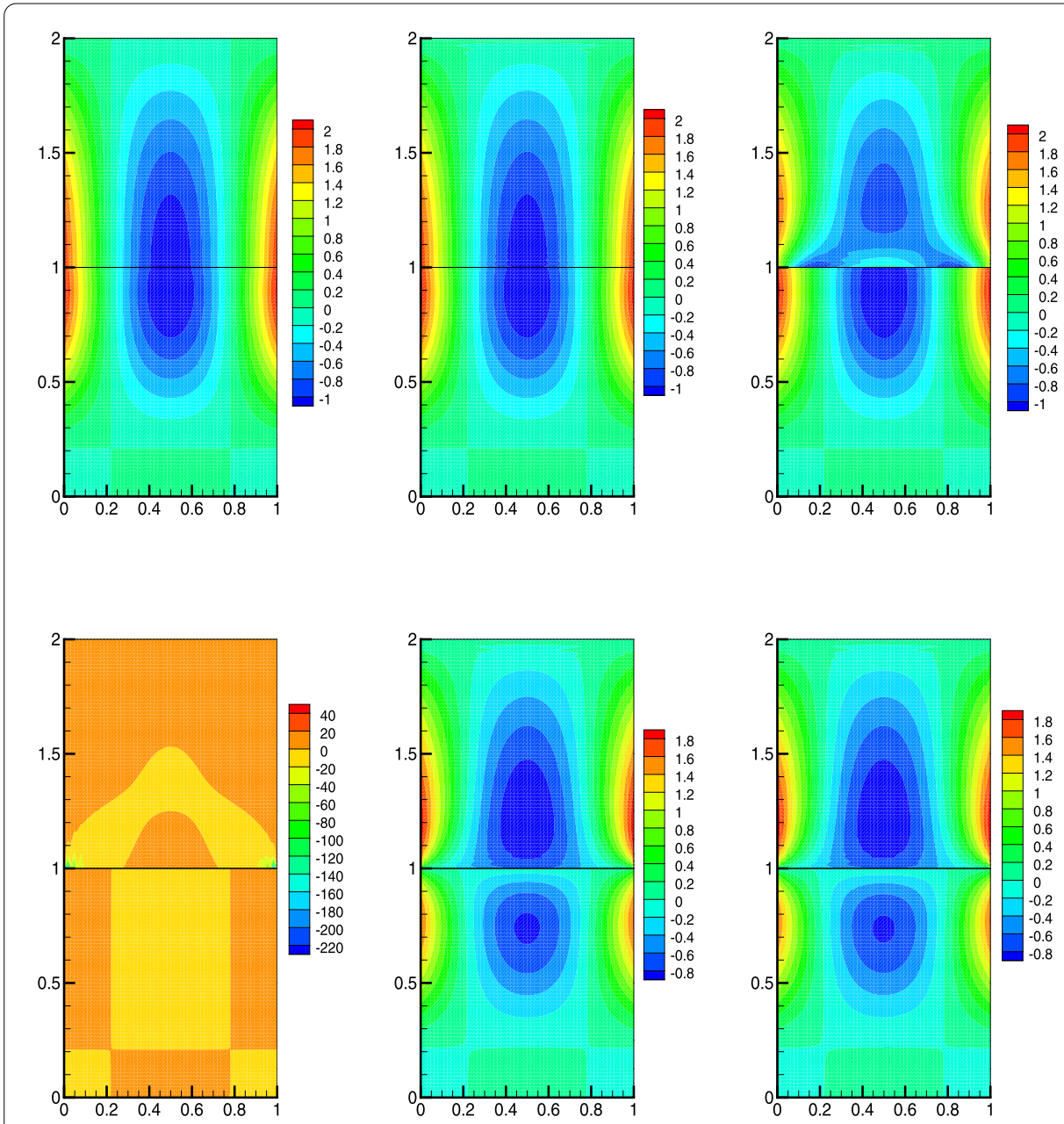

Figure 4 An illustration of the pressure contour for the true solution and approximate solution with the different values of the stabilization parameter: true solution (top-left); $\delta=0.1$ (top-middle); $\delta=1$ (top-right); $\delta=1000$ (down-left); $\delta=0.0001$ (down-middle); $\delta=0$ (down-right)

Table 1 Approximate error of the stabilized finite element scheme for test 1

\begin{tabular}{lllllll}
\hline $\mathrm{h}$ & $\left\|\mathbf{u}_{f}^{h}-\mathbf{u}_{f}\right\|_{0, \Omega_{f}}$ & $\left\|\mathbf{u}_{f}^{h}-\mathbf{u}_{f}\right\|_{1, \Omega_{f}}$ & $\left\|p^{h}-p\right\|_{0, \Omega_{f}}$ & $\left\|\phi^{h}-\phi\right\|_{0, \Omega_{p}}$ & $\left\|\phi^{h}-\phi\right\|_{1, \Omega_{p}}$ & $\left\|\mathbf{u}_{p}^{h}-\mathbf{u}_{p}\right\|_{0, \Omega_{p}}$ \\
\hline $1 / 4$ & 0.06698400 & 0.2051800 & 0.9868740 & 0.25280000 & 0.5592480 & 0.11349000 \\
$1 / 8$ & 0.01689870 & 0.1022890 & 0.2969910 & 0.03583200 & 0.1964390 & 0.02378000 \\
$1 / 16$ & 0.00423272 & 0.0510305 & 0.0972678 & 0.00861098 & 0.0957356 & 0.00583354 \\
$1 / 32$ & 0.00105840 & 0.0254780 & 0.0332221 & 0.00213774 & 0.0475719 & 0.00145604 \\
$1 / 64$ & 0.00026471 & 0.0127283 & 0.0115640 & 0.00051766 & 0.0237599 & 0.00036354 \\
\hline
\end{tabular}

to consider the value of the stabilization parameter $\delta=0.1$ to perform the numerical tests which ensure an optimal convergence order and generate velocity and pressure contours accurately.

To demonstrate the order of convergence of the finite element stabilized scheme, we introduce Table 1, the errors between the computed and exact solutions with varying spacing $h=1 / 4,1 / 8,1 / 16,1 / 32,1 / 64$. Figure 5 is the $\log$ - $\log$ plot of the data in Table 1 . We can observe from the figures that the optimal convergence order is obtained, which supports the theoretical analysis. 

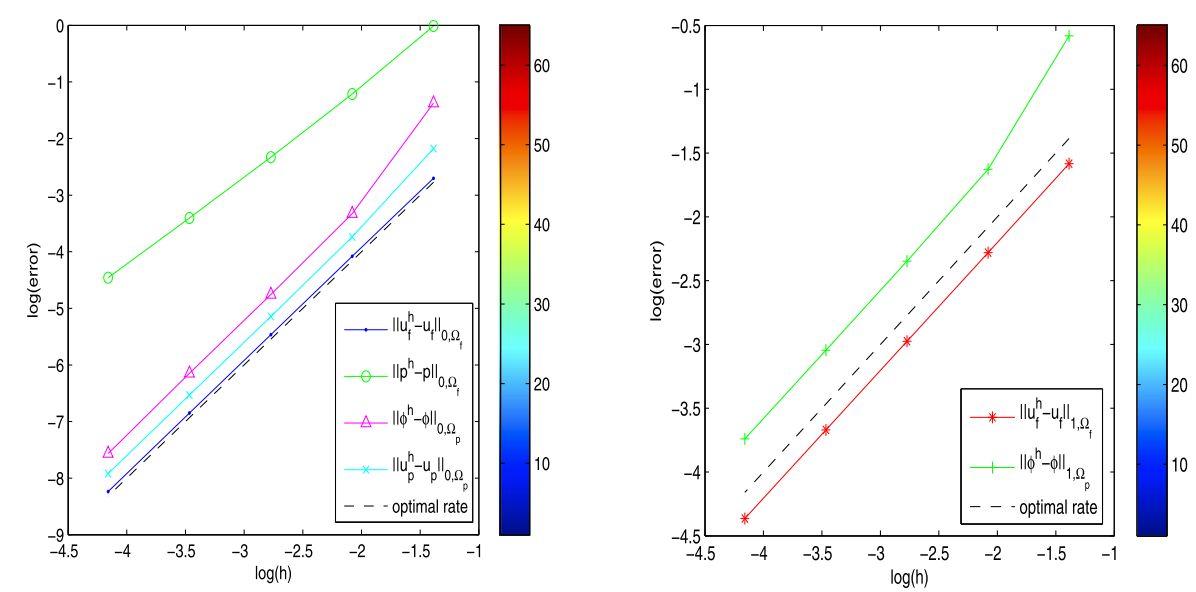

Figure 5 The order of convergence of $L^{2}$-norm (left) and $H^{1}$-norm (right) for the change on the convergence order of the stabilized finite element scheme for test 1

\subsection{Convergence test 2}

The main purpose of the second numerical is to show the influence of the physical parameter hydraulic conductivity $\mathbf{K}$ on the convergence order inspired by [33] for the stabilized finite element scheme. In this experiment, we set all the values of the physical parameters $\nu, \rho, g, \mathbf{K}, \alpha, \delta$ the same as in the previous computation, except for different values of the hydraulic conductivity parameter $\mathbf{K}$ where $\mathbf{K}=k \mathbb{I}, k=0.1,0.01,0.001$.

The exact solution for the second numerical test satisfying the Beavers-Joseph-Saffman interface conditions is given by

$$
\left\{\begin{array}{l}
\mathbf{u}_{f}=\left(\begin{array}{c}
\left(y^{2}-2 y+1\right) \\
\left(x^{2}-x\right)
\end{array}\right), \\
p=\left[2 v(x+y-1)+\frac{g n}{3 k}\right], \\
\phi=\left[\frac{n}{k}\left(x(1-x)(y-1)+\frac{1}{3} y^{3}-y^{2}+y\right)+\frac{2 v}{g} x\right], \\
\mathbf{u}_{p}=-\mathbf{K} \nabla \phi .
\end{array}\right.
$$

The Dirichlet boundary condition and source terms of the model problem are chosen in such a way that the above-listed functions are the exact solutions of the model problem.

In Tables 2, 3 and 4, the approximation errors in differential norms are listed for the stabilized finite element scheme with varying hydraulic conductivity $\mathbf{K}=0.1 \mathbb{I}, 0.01 \mathbb{I}$, $0.001 I$. From these tables we observe that the order of the magnitude of the Stokes and Darcy pressure $p$ and $\phi$ gradually increases with the decrease of the value of the parameter K. From Fig. 6, we can see that the optimal convergence order is also obtained, which confirms the theoretical analysis.

\section{Conclusion}

In this contribution, we investigated a new fully mixed finite element method to solve the Stokes-Darcy fluid flow model without introducing any Lagrange multiplier. We proposed a stabilized finite element scheme and introduced a stabilization term to ensure the wellposedness of the temporal discretization. The desired stability and convergence analysis, 
Table 2 Approximate error of the stabilized finite scheme for test 2 with $\mathbf{K}=0.1 \mathbb{I}$

\begin{tabular}{lllllll}
\hline $\mathrm{h}$ & $\left\|\mathbf{u}_{f}^{h}-\mathbf{u}_{f}\right\|_{0, \Omega_{f}}$ & $\left\|\mathbf{u}_{f}^{h}-\mathbf{u}_{f}\right\|_{1, \Omega_{f}}$ & $\left\|p^{h}-p\right\|_{0, \Omega_{f}}$ & $\left\|\phi^{h}-\phi\right\|_{0, \Omega_{p}}$ & $\left\|\phi^{h}-\phi\right\|_{1, \Omega_{p}}$ & $\left\|\mathbf{u}_{p}^{h}-\mathbf{u}_{p}\right\|_{0, \Omega_{p}}$ \\
\hline $1 / 4$ & 0.0161660000 & 0.1895230 & 0.05247790 & 0.048723100 & 1.4312000 & 0.0205940000 \\
$1 / 8$ & 0.0040720000 & 0.0944033 & 0.01628890 & 0.011688300 & 0.7203930 & 0.0054969900 \\
$1 / 16$ & 0.0010201200 & 0.0471172 & 0.00525163 & 0.002889210 & 0.3606690 & 0.0014169300 \\
$1 / 32$ & 0.0002548480 & 0.0235401 & 0.00174311 & 0.000721144 & 0.1804010 & 0.0003594990 \\
$1 / 64$ & $6.37027 \mathrm{e}-005$ & 0.0117660 & 0.00059351 & 0.000180326 & 0.0902083 & $9.05304 \mathrm{e}-005$ \\
\hline
\end{tabular}

Table 3 Approximate error of the stabilized finite element scheme for test 2 with $\mathbf{K}=0.01 \mathbb{I}$

\begin{tabular}{lllllcl}
\hline $\mathrm{h}$ & $\left\|\mathbf{u}_{f}^{h}-\mathbf{u}_{f}\right\|_{0, \Omega_{f}}$ & $\left\|\mathbf{u}_{f}^{h}-\mathbf{u}_{f}\right\|_{1, \Omega_{f}}$ & $\left\|p^{h}-p\right\|_{0, \Omega_{f}}$ & $\left\|\phi^{h}-\phi\right\|_{0, \Omega_{p}}$ & $\left\|\phi^{h}-\phi\right\|_{1, \Omega_{p}}$ & $\left\|\mathbf{u}_{p}^{h}-\mathbf{u}_{p}\right\|_{0, \Omega_{p}}$ \\
\hline $1 / 4$ & 0.0165941000 & 0.1898900 & 0.09290030 & 0.46289000 & 14.34060 & 0.0215030000 \\
$1 / 8$ & 0.0046533600 & 0.0948421 & 0.02580190 & 0.11471700 & 7.207800 & 0.0056623600 \\
$1 / 16$ & 0.0012938400 & 0.0472456 & 0.00675441 & 0.02864350 & 3.606840 & 0.0014376000 \\
$1 / 32$ & 0.0003417120 & 0.0235623 & 0.00199893 & 0.00718127 & 1.804010 & 0.0003616240 \\
$1 / 64$ & $8.73879 \mathrm{e}-005$ & 0.0117691 & 0.00063884 & 0.00179964 & 0.902083 & $9.07503 \mathrm{e}-005$ \\
\hline
\end{tabular}

Table 4 Approximate error of the stabilized finite element scheme for test 2 with $\mathbf{K}=0.001 \mathbb{I}$

\begin{tabular}{lllllcl}
\hline $\mathrm{h}$ & $\left\|\mathbf{u}_{f}^{h}-\mathbf{u}_{f}\right\|_{0, \Omega_{f}}$ & $\left\|\mathbf{u}_{f}^{h}-\mathbf{u}_{f}\right\|_{1, \Omega_{f}}$ & $\left\|p^{h}-p\right\|_{0, \Omega_{f}}$ & $\left\|\phi^{h}-\phi\right\|_{0, \Omega_{p}}$ & $\left\|\phi^{h}-\phi\right\|_{1, \Omega_{p}}$ & $\left\|\mathbf{u}_{p}^{h}-\mathbf{u}_{p}\right\|_{0, \Omega_{p}}$ \\
\hline $1 / 4$ & 0.016845100 & 0.1902420 & 0.12546000 & 4.5981200 & 143.695 & 0.022608400 \\
$1 / 8$ & 0.005788180 & 0.0964517 & 0.05396490 & 1.1318200 & 72.1796 & 0.006306740 \\
$1 / 16$ & 0.002560890 & 0.0488275 & 0.01943480 & 0.2813460 & 36.0814 & 0.001683910 \\
$1 / 32$ & 0.001052120 & 0.0242530 & 0.00616251 & 0.0705812 & 18.0412 & 0.000422170 \\
$1 / 64$ & 0.000358495 & 0.0119415 & 0.00191977 & 0.0177721 & 9.02091 & 0.000101925 \\
\hline
\end{tabular}

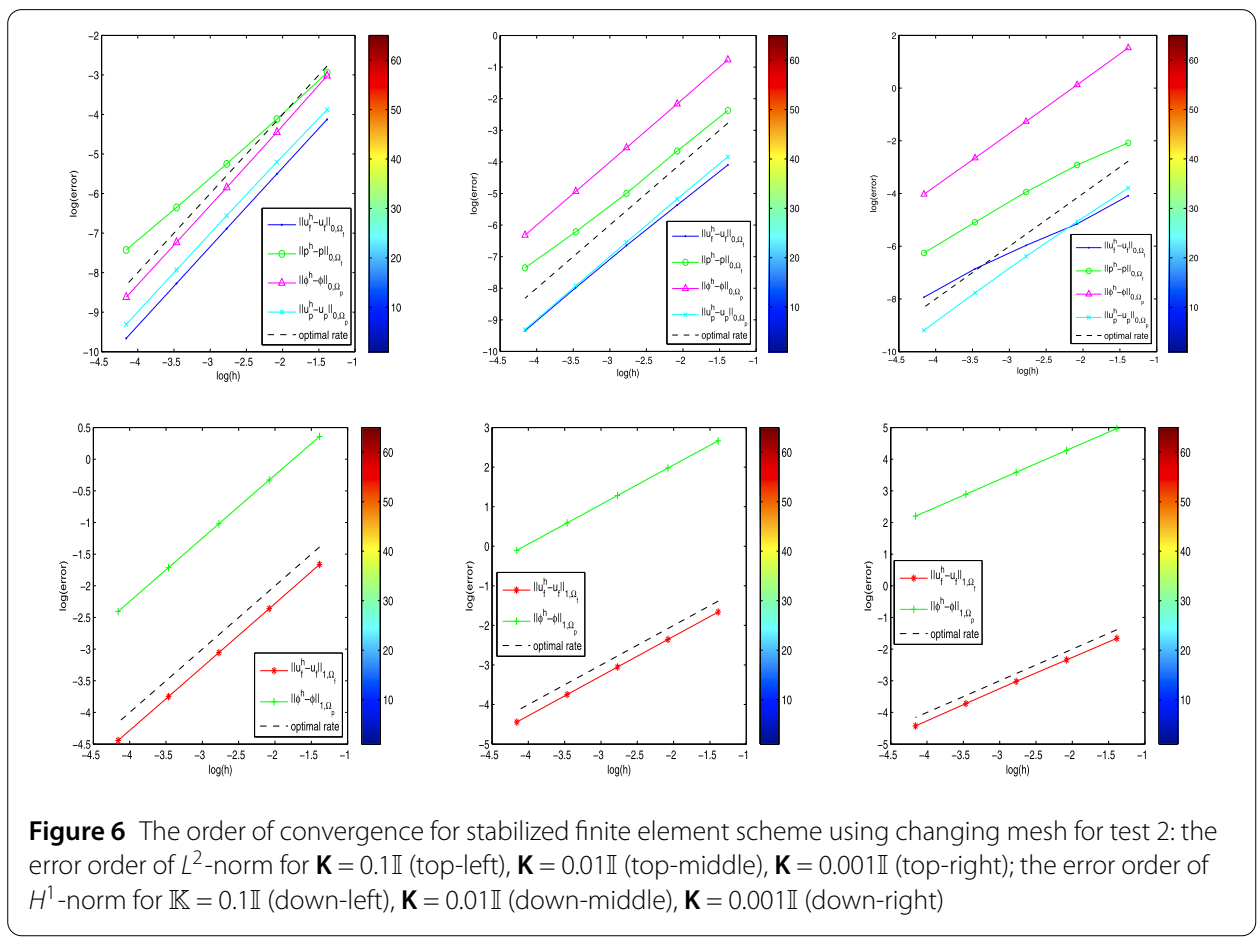

including optimal error estimates, was derived for the proposed algorithm. To show the exclusive feature of the finite element scheme and numerical methods, we performed two numerical tests and illustrated the results of the experiments. The numerical test revealed the accuracy and efficiency of the proposed mixed finite element method. 


\section{Funding}

This work was supported by the NSF of China with Grant Nos 11501097, 11471071 and 11571115 , Foundation Research Project of Shenzhen with Grant Nos ZDSYS201707280904031 and JCYJ20160817172025986, and Science and Technology Commission of Shanghai Municipality with Grant No. 18dz2271000.

\section{Availability of data and materials}

Please contact the authors for data requests.

\section{Competing interests}

The authors declare that they have no competing interests.

\section{Authors' contributions}

The study was carried out in collaboration among all authors. All authors read and approved the final manuscript.

\section{Author details}

${ }^{1}$ School of Science, Donghua University, Shanghai, China. ${ }^{2}$ School of Mathematical Sciences, East China Normal University, Shanghai, China. ${ }^{3}$ Shanghai Key Laboratory of Pure Mathematics and Mathematical Practice, Shanghai, China. ${ }^{4}$ College of Science, Harbin Institute of Technology, Shenzhen, China.

\section{Publisher's Note}

Springer Nature remains neutral with regard to jurisdictional claims in published maps and institutional affiliations.

\section{Received: 20 July 2018 Accepted: 18 September 2018 Published online: 29 September 2018}

\section{References}

1. Cao, Y., Gunzburger, M., He, X.M., Wang, X.: Parallel, non-iterative, multi-physics domain decomposition methods for time-dependent Stokes-Darcy systems. Math. Comput. 83, 1617-1644 (2014)

2. Boubendir, Y., Tlupova, S.: Domain decomposition methods for solving Stokes-Darcy problems with boundary integrals. SIAM J. Sci. Comput. 35, B82-B106 (2013)

3. Cai, M., Mu, M., Xu, J.: Numerical solution to a mixed Navier-Stokes/Darcy model by the two-grid approach. SIAM J. Numer. Anal. 47, 3325-3338 (2009)

4. Cao, Y., Gunzburger, M., He, X.M., Wang, X.: Robin-Robin domain decomposition methods for the steady-state Stokes-Darcy system with Beaver-Joseph interface condition. Numer. Math. 117, 601-629 (2011)

5. Vassilev, D., Yotov, I.: Coupling Stokes-Darcy flow with transport. SIAM J. Sci. Comput. 31, 3661-3684 (2009)

6. Beavers, G., Joseph, D.: Boundary conditions at a naturally permeable wall. J. Fluid Mech. 30, 197-207 (1967)

7. Saffman, P.: On the boundary condition at the surface of a porous medium. Stud. Appl. Math. 50, 93-101 (1971)

8. Jones, I.P.: Low Reynolds number flow past a porous spherical shell. Proc. Camb. Philol. Soc. 73, 231-238 (1973)

9. Gartling, D.K., Hickox, C.E., Givler, R.C.: Simulation of coupled viscous and porous flow problems. Int. J. Comput. Fluid Dyn. 7, 23-48 (1996)

10. Salinger, A.G., Aris, R., Derby, J.J.: Finite element formulations for large-scale, coupled flows in adjacent porous and open fluid domains. Int. J. Numer. Methods Fluids 18, 1185-1209 (1994)

11. Layton, W.J., Schieweck, F., Yotov, I.: Coupling fluid flow with porous media flow. SIAM J. Numer. Anal. 40, 2195-2218 (2003)

12. Discacciati, M., Miglio, E., Quarteroni, A.: Mathematical and numerical models for coupling surface and groundwater flows. Appl. Numer. Math. 43, 57-74 (2002)

13. Jäger, W., Mikelić, M.: On the interface boundary condition of Beavers, Joseph, and Saffman. SIAM J. Appl. Math. 60, $1111-1127(2000)$

14. Payne, L.E., Straughan, B.: Analysis of the boundary condition at the interface between a viscous fluid and a porous medium and related modeling questions. J. Math. Pures Appl. 77, 317-354 (1998)

15. Arbogast, T., Brunson, D.S.: A computational method for approximating a Darcy-Stokes system governing a vuggy porous medium. Comput. Geosci. 11, 207-218 (2007)

16. Badea, L., Discacciati, M., Quarteroni, A.: Numerical analysis of the Navier-Stokes/Darcy coupling. Numer. Math. 115 $195-227$ (2010)

17. Cao, Y., Gunzburger, M., Hu, X., Hua, F., Wang, X., Zhao, W.: Finite element approximations for Stokes-Darcy flow with Beavers-Joseph interface conditions. SIAM J. Numer. Anal. 47, 4239-4256 (2010)

18. Ervin, V.J., Jenkins, E.W., Sun, S.: Coupled generalized nonlinear Stokes flow with flow through a porous medium. SIAM J. Numer. Anal. 47, 929-952 (2009)

19. Cao, Y., Gunzburger, M., Hu, X., Hua, F., Wang, X.: Coupled Stokes-Darcy model with Beavers-Joseph interface boundary condition. Commun. Math. Sci. 8, 1-25 (2010)

20. Karper, T., Mardal, K.A., Winther, R.: Unified finite element discretizations of coupled Darcy-Stokes flow. Numer. Methods Partial Differ. Equ. 25, 311-326 (2009)

21. Rui, H., Zhang, R.: A unified stabilized mixed finite element method for coupling Stokes and Darcy flows. Comput. Methods Appl. Mech. Eng. 198, 33-36 (2009)

22. Mu, M., Xu, J.: A two-grid method of a mixed Stokes-Darcy model for coupling fluid flow with porous media flow. SIAM J. Numer. Anal. 45, 1801-1813 (2007)

23. Cai, M., Mu, M., Xu, J.: Numerical solution to a mixed Navier-Stokes/Darcy model by the two-grid approach. SIAM J. Numer. Anal. 47, 3325-3338 (2009)

24. Cai, M.M.: A multilevel decoupled method for a mixed Stokes/Darcy model. J. Comput. Appl. Math. 236, 2452-2465 (2012)

25. Zuo, L., Hou, Y.: A decoupling two-grid algorithm for the mixed Stokes-Darcy model with the Beavers-Joseph interface condition. Numer. Methods Partial Differ. Equ. 30, 1066-1082 (2014) 
26. Hou, Y.: Optimal error estimates of a decoupled scheme based on two-grid finite element for mixed Stokes-Darcy model. Appl. Math. Lett. 57, 90-96 (2016)

27. Zuo, L., Hou, Y:: A two-grid decoupling method for the mixed Stokes-Darcy model. J. Comput. Appl. Math. 275, 139-147 (2015)

28. Zuo, L., Hou, Y.: Numerical analysis for the mixed Navier-Stokes and Darcy problem with the Beavers-Joseph interface condition. Numer. Methods Partial Differ. Equ. 31, 1009-1030 (2015)

29. Girault, V., Rivière, B.: DG approximation of coupled Navier-Stokes and Darcy equations by Beaver-Joseph-Saffman interface condition. SIAM J. Numer. Anal. 47, 2052-2089 (2009)

30. Rivière, B.: Analysis of a discontinuous finite element method for coupled Stokes and Darcy problems. J. Sci. Comput. 22, 479-500 (2005)

31. Rivière, B., Yotov, I.: Locally conservative coupling of Stokes and Darcy flows. SIAM J. Numer. Anal. 42, 1959-1977 (2005)

32. Lipnikov, K., Vassilev, D., Yotov, I.: Discontinuous Galerkin and mimetic finite difference methods for coupled Stokes-Darcy flows on polygonal and polyhedral grids. Numer. Math. 126, 321-360 (2014)

33. $\mathrm{Mu}, \mathrm{M}$. , Zhu, X.: Decoupled schemes for a non-stationary mixed Stokes-Darcy model. Math. Comput. 79, 707-731 (2010)

34. Shan, L., Zhang, Y.: Error estimates of the partitioned time stepping method for the evolutionary Stokes-Darcy flows. Comput. Math. Appl. 73, 713-726 (2017)

35. Shan, L., Zheng, H.: Partitioned time stepping method for fully evolutionary Stokes-Darcy flow with Beavers-Joseph interface conditions. SIAM J. Numer. Anal. 51, 813-839 (2013)

36. Shan, L., Zheng, H., Layton, W.J: A decoupling method with different subdomain time steps for the nonstationary Stokes-Darcy model. Numer. Methods Partial Differ. Equ. 29, 549-583 (2013)

37. Urquiza, J.M., N'Dri, D., Garon, A., Delfour, M.C.: Coupling Stokes and Darcy equations. Appl. Numer. Math. 58, 525-538 (2008)

38. Ervin, V.J., Jenkins, E.M., Lee, H.: Approximation of the Stokes-Darcy system by optimization. J. Sci. Comput. 59, 775-794 (2014)

39. Lee, H., Rife, K.: Least squares approach for the time-dependent nonlinear Stokes-Darcy flow. Comput. Math. Appl. 67, 1806-1815 (2014)

40. Discacciati, M., Quarteroni, A., Valli, A.: Robin-Robin domain decomposition methods for the Stokes-Darcy coupling. SIAM J. Numer. Anal. 45, 1246-1268 (2007)

41. He, X.M., Li, J., Lin, Y.P., Ming, J.: A domain decomposition method for the steady-state Navier-Stokes-Darcy mode with the Beavers-Joseph interface condition. SIAM J. Sci. Comput. 37, S264-S290 (2015)

42. Discacciati, M.: Iterative methods for Stokes/Darcy coupling. In: Domain Decomposition Methods in Science and Engineering. Lect. Notes Comput. Sci. Eng., vol. 40, pp. 563-570. Springer, Berlin (2005)

43. Discacciati, M., Quarteroni, A.: Convergence analysis of a subdomain iterative method for the finite element approximation of the coupling of Stokes and Darcy equations. Comput. Vis. Sci. 6, 93-103 (2004)

44. Vassilev, D., Wang, C., Yotov, I.: Domain decomposition for coupled Stokes and Darcy flows. Comput. Methods Appl. Mech. Eng. 268, 264-283 (2014)

45. Jiang, B.: A parallel domain decomposition method for coupling of surface and groundwater flows. Comput. Methods Appl. Mech. Eng. 198, 947-957 (2009)

46. Feng, W., He, X.M., Wang, Z., Zhang, X.: Non-iterative domain decomposition methods for a non-stationary Stokes-Darcy model with Beavers-Joseph interface condition. Appl. Math. Comput. 219, 453-463 (2012)

47. Discacciati, M., Quarteroni, A.: Analysis of a domain decomposition method for the coupling of Stokes and Darcy equations. In: Numerical Mathematics and Advanced Applications, pp. 3-20. Springer, Milan (2003)

48. Chen, W., Gunzburger, M., Hua, F., Wang, X.: A parallel Robin-Robin domain decomposition method for the Stokes-Darcy system. SIAM J. Numer. Anal. 49, 1064-1084 (2011)

49. Du, G., Zuo, L.: Local and parallel finite element method for the mixed Navier-Stokes/Darcy model with Beavers-Joseph interface conditions. Acta Math. Sci. 37B, 1331-1347 (2017)

50. Markus, S., Houstis, E., Catlin, A., Rice, J., Tsompanopoulou, P., Vavalis, E., Gottfried, D., Su, K., Balakrishnan, G.: An agent-based netcentric framework for multidisciplinary problem solving environments (MPSE). Int. J. Comput. Eng. Sci. 1, 33-60 (2000)

51. Mu, M.: Solving composite problems with interface relaxation. SIAM J. Sci. Comput. 20, 1394-1416 (1999)

52. Girault, V., Vassilev, D., Yotov, I.: Mortar multiscale finite element methods for Stokes-Darcy flows. Numer. Math. 127, 93-165 (2014)

53. Ervin, V.J., Jenkins, E.W., Sun, S.: Coupling nonlinear Stokes and Darcy flow using mortar finite elements. Appl. Numer. Math. 61, 1198-1222 (2011)

54. Babuŝkka, I., Gatica, G.N.: A residual-based a posteriori error estimator for the Stokes-Darcy coupled problem. SIAM J. Numer. Anal. 48, 498-523 (2010)

55. Gatica, G.N., Oyarzúa, R., Sayas, F.J.: A residual-based a posteriori error estimator for a fully-mixed formulation of the Stokes-Darcy coupled problem. Comput. Methods Appl. Mech. Eng. 200, 1877-1891 (2011)

56. Huang, P., Chen, J., Cai, M.: A mixed and nonconforming FEM with nonmatching meshes for a coupled Stokes-Darcy model. J. Sci. Comput. 53, 377-394 (2012)

57. Gatica, G.N., Oyarzúa, R., Sayas, F.J.: A conforming mixed finite-element method for the coupling of fluid flow with porous media flow. IMA J. Numer. Anal. 29, 86-108 (2009)

58. Gatica, G.N., Oyarzúa, R., Sayas, F.J.: Convergence of a family of Galerkin discretizations for the Stokes-Darcy coupled problem. Numer. Methods Partial Differ. Equ. 27, 721-748 (2011)

59. Gatica, G.N., Oyarzúa, R., Sayas, F.J.: Analysis of fully-mixed finite element methods for the Stokes-Darcy coupled problem. Math. Comput. 80, 1911-1948 (2011)

60. Burman, E., Hansbo, P.: A unified stabilized method for Stokes' and Darcy's equations. J. Comput. Appl. Math. 198 35-51 (2007)

61. Cai, M., Mu, M. Xu, J.: Preconditioning techniques for mixed Stokes/Darcy model in porous media applications. J. Comput. Appl. Math. 233, 346-355 (2009) 
62. Camaño, J., Gatica, G.N., Oyarzúa, R., Ruiz-Baier, R., Venegas, P.: New fully-mixed finite element methods for the Stokes-Darcy coupling. Comput. Methods Appl. Mech. Eng. 295, 362-395 (2015)

63. Figueroa, L., Gatica, G.N., Márquez, A.: Augmented mixed finite element methods for the stationary Stokes equations. SIAM J. Sci. Comput. 31, 1082-1119 (2008)

64. Carstensen, C.: A posteriori error estimate for the mixed finite element method. Math. Comput. 66, 465-476 (1997)

65. Brezzi, F., Fortin, M.: Mixed and Hybrid Finite Element Methods. Springer Series in Computational Mathematics, vol. 15. Springer, New York (1991)

66. Brezzi, F., Douglas, J. Jr., Fortin, M., Marini, L.D.: Efficient rectangular mixed finite elements in two and three space variables. Math. Model. Anal. 21, 581-604 (1987)

67. Adams, R.A., Fournier, J.J.F., Sobolev Spaces. 2nd ed., Pure Appl. Math. (Amst.), 140, Elsevier, Amsterdam (2003)

68. Arnold, D.N., Brezzi, F., Fortin, M.: A stable finite element for the Stokes equations. Calcolo 21, 337-344 (1984)

69. Brezzi, F., Douglas, J. Jr., Marini, L.D.: Two families of mixed finite elements for second order elliptic problems. Numer. Math. 47, 217-235 (1985)

70. Hecht, F., Le Hyaric, A., Ohtsuka, K., Pironneau, O.: Freefem++, Finite elements software. http://www.freefem.org/ff++/

\section{Submit your manuscript to a SpringerOpen ${ }^{\circ}$ journal and benefit from:}

- Convenient online submission

- Rigorous peer review

- Open access: articles freely available online

- High visibility within the field

- Retaining the copyright to your article

Submit your next manuscript at $\boldsymbol{~ s p r i n g e r o p e n . c o m ~}$ 